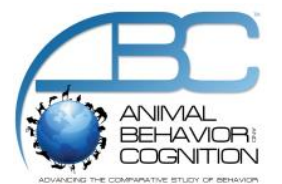

\title{
Primates' Food Preferences Predict Their Food Choices Even Under Uncertain Conditions
}

\author{
Sarah M. Huskisson ${ }^{1}$, Crystal L. Egelkamp ${ }^{1}$, Sarah L. Jacobson ${ }^{1,2}$, Stephen R. Ross ${ }^{1}$, \\ \& Lydia M. Hopper ${ }^{*}$
}

\author{
${ }^{1}$ Lester E. Fisher Center for the Study and Conservation of Apes, Lincoln Park Zoo, Chicago, IL, USA \\ 2 The Graduate Center, City University of New York, New York, NY, USA \\ *Corresponding author (Email: lhopper@lpzoo.org)
}

Citation - Huskisson, S. M., Egelkamp, C. L., Jacobson, S. L., Ross, S. R., \& Hopper, L. M. (2021). Primates' food preferences predict their food choices even under uncertain conditions. Animal Behavior and Cognition, 8(1), 69-96. https://doi.org/10.26451/abc.08.01.06.2021

\begin{abstract}
Primates' food preferences are typically assessed under conditions of certainty. To increase ecological validity, and to explore primates' decision making from a comparative perspective, we tested three primate species (Pan troglodytes, Gorilla gorilla gorilla, Macaca fuscata) $(N=18)$ in two food-preference tests that created different conditions of uncertainty. In the first, we showed subjects pairs of photographs of six foods in a randomized manner within each session, so subjects could not predict the next pairing and had to respond in accordance with their preferences. We found individual differences in subjects' preference and differences in six subjects' preferences when comparing their selections in this test to selections made when trials were blocked by food pairing (tested previously: Huskisson et al., 2020). In each trial of the second test we paired the food stimuli with a 'chance' symbol, representing a random reward of one of the six foods. Across species, each subject's propensity to select chance over the known food varied by food type: when the known option was highly preferred, subjects were less likely to select the chance symbol. Additionally, $61.11 \%$ of the subjects' rates of selecting foods in the first test showed a positive trend with the rates of selecting the same foods under conditions of uncertainty here; three of these subjects' selection rates were significantly correlated between tests. Finally, if the food chimpanzees received for selecting the chance symbol was a preferred food, they were more likely to select chance again in the subsequent trial.
\end{abstract}

Keywords - Chance, Chimpanzee, Food preference, Gorilla, Japanese macaque, Welfare

Virtually all wild primates live in dynamic environments and often must make split-second decisions about different options, particularly with regard to their food choices. A combination of intrinsic and extrinsic factors can shape individual animals' preferences. Primates' food choices may be dictated by their relative preference for a food item compared to what others are available (e.g., Freeman et al., 2013), the respective densities of food items that are present (e.g., Parrish et al., 2020), the nutritional content of the food (e.g., Hohmann et al., 2010; Verspeek \& Stevens, 2020), and/or how far an animal is willing to go (in terms of energy expenditure, social conflict, etc.) to obtain a certain resource (Bonnie et al., 2019; Bramlett et al., 2012; Hopper et al., 2015; Schwartz et al., 2016). Furthermore, wild primates often have to make decisions based on incomplete information (De Petrillo \& Rosati, 2019). Experimental research investigating the influence of how choices are framed can help elucidate primates' risk aversion and decision-making strategies (Lakshminarayanan et al., 2011; Proctor et al., 2014). Even when relatively complete information is provided, individuals' choices may be affected by the presence of a "decoy," or distractor element that pulls a subject's attention away from the target (and potentially better) options (e.g., Sanchez-Amaro et al., 2019).

While primates, including humans, are typically risk averse, their likelihood to show chance-taking behavior is mediated by the relative risk/reward payoffs (Haun et al., 2011) and how the opportunities are 
presented (Heilbronner \& Hayden, 2016). To date, however, most research into primate risk taking has focused on only a few taxa (notably, Pan and Sapajus, De Petrillo \& Rosati, 2019), with less attention given to other species. We wanted to remedy this by studying two lesser-studied species (Gorilla gorilla gorilla and Macaca fuscata) and compare their responses with more commonly-studied chimpanzees (Pan troglodytes) in two tests of food preference, which created different conditions of uncertainty. While gorillas maintain a constant diet of low-energy vegetation (Rogers et al., 2004), chimpanzees and Japanese macaques are omnivorous and take advantage of high-value, high-energy items to eat (Moscovice et al., 2007; Tsuji et al., 2008). Considering this, we might expect chimpanzees and macaques to be more willing to take chances to obtain a potentially higher-quality reward than gorillas (Leinwand et al., 2020).

In typical tests of primates' food preferences (e.g., Huskisson et al., 2020; Vinyard et al., 2016), as well as in tests of decision making and risk aversion (e.g., Brosnan et al., 2015; Leinwand et al., 2020; Proctor et al., 2014), trials are presented in a blocked manner within condition so subjects are not required to make new choices with each trial. However, it is perhaps more ecologically valid to ask questions pertaining to primates' food choices and preferences when subjects are faced with trial-by-trial changes with respect to the relative food values available, simulating the decision- making challenges their wild counterparts face when considering the uncertainty in future food availability. Therefore, and building upon our previous research testing primates' preferences using touchscreens (Hopper et al., 2019; Huskisson et al., 2020), we presented primates with food preference tests under two conditions of uncertainty. Expanding upon a typical forced-choice preference test, in the first phase of our study (Phase 1) we presented subjects with pairs of photographs of two different foods that varied across trials within each testing session. This random presentation style made it more difficult for subjects to predict the next food pairing, and thus subjects should vary their choices from trial to trial as they adjust their evaluations of the available foods and their relative value to the subject. In other words, the random presentation of food options creates a degree of uncertainty in the choices that the subjects must make (e.g., Gray et al., 2018). Such uncertainty buffers against a tendency for subjects to simply select the same food items repeatedly as they need to weigh their relative preferences for each of the two foods offered in every trial. Furthermore, in randomized presentation, subjects are making choices that are in the moment in response to "fresh" trials, as opposed to responding to repeated trials presenting the same stimuli.

While the food options that subjects could choose between varied across trials in Phase 1 , the subjects always had complete information within each trial as to their options (i.e., they knew exactly which two foods they had to choose from). What if they had incomplete information, which resulted in some uncertainty about what options they should select? In such cases, primates have been observed to select a "sure bet," even if is a smaller reward, as opposed to taking advantage of an uncertain option that may yield them a larger reward (e.g., Farashahi et al., 2019; Haun et al., 2011; Rivière et al., 2019). Therefore, in the second phase of our study (Phase 2), we evaluated subjects' responses under conditions of greater uncertainty. Specifically, we showed subjects a "known" choice (a photograph of one of the six foods presented in Phase 1) and an "unknown" choice (a question mark stimulus representing a payoff of one of the six foods at random but equal probability). In these trials, the subject could either choose the known reward or take a chance and receive one of six foods, some of which were preferred, and some of which were not preferred (both in absolute terms and relative to the known option). In doing this, we hoped to simulate ecological contexts in which resources are constantly fluctuating, and there were no fixed options from one choice to the next.

From our previous touchscreen-based assessments of these primates' food preferences (Huskisson et al., 2020), we hypothesized that subjects would exhibit distinct food preferences. We had previously tested the subjects' preferences by presenting pairs of foods in a blocked presentation (i.e., 90 trials per pairing) to increase certainty regarding the available options within sessions and across trials. In the present study (Phase 1), we varied the pairs of foods presented across trials, which encouraged subjects to more carefully consider their choices. We did not have a directional prediction as to whether this methodological modification would alter the subjects' choices and revealed preferences. However, for our second phase, in which subjects chose between an unknown and a known food option, we predicted that subjects would be more likely to select the known, "safe" choice when it was a preferred food, but that they would be more likely to take a chance when the known food option was of low value. Specifically, we predicted that the 
rate at which subjects selected specific known food options in Phase 2 would correlate with the rate at which they selected those same foods in Phase 1. Given the feeding ecology hypothesis (Heilbronner et al., 2008), we predicted that, across food pairings, macaques and chimpanzees would be more likely than gorillas to select the unknown option (i.e., would be more likely to take a risk) (see also Leinwand et al., 2020). In trials where subjects selected the chance symbol in Phase 2, we evaluated whether the value of the reward they received for doing so (in absolute and relative terms and as compared to the known, pictured food) impacted their propensity to select chance in the following trial. We predicted that subjects would be more likely to select chance in the next trial if the unknown reward they received was more highly valued than if it was of a lower or similar value to the known item. Understanding the motivation behind choices, in terms of food value, can provide an important context to how primates view and value certain foods, which is important both for understanding the mechanisms that may underlie primate feeding ecology and for the application in the daily husbandry of captive primates.

\section{Method}

\section{Ethics}

This study was approved by the Lincoln Park Zoo Research Committee and was conducted in accordance with the American Society of Primatologists' Principles for the Ethical Treatment of Nonhuman Primates. Lincoln Park Zoo animal care, veterinary, and nutrition staff approved all foods prior to commencement of research, and all foods were a regular part of subjects' diets (Table 1). We did not make any modifications to animal husbandry schedules.

\section{Table 1}

Typical Uses of each Food Item Outside of Preference Testing

\begin{tabular}{|c|c|c|c|}
\hline & Food & Frequency in Diets & Nutritional Content (per 1 cup) \\
\hline \multicolumn{4}{|l|}{ Apes } \\
\hline & Apple & Several times a week & $.2 \mathrm{~g}$ fat, $17 \mathrm{~g}$ carbs, $13 \mathrm{~g}$ sugar \\
\hline & Carrot & Several times a week & $.3 \mathrm{~g}$ fat, $12 \mathrm{~g}$ carbs, $6.1 \mathrm{~g}$ sugar \\
\hline & Cucumber & Several times a week & $.2 \mathrm{~g}$ fat, $3.8 \mathrm{~g}$ carbs, $1.8 \mathrm{~g}$ sugar \\
\hline & Grape & Several times a week & $.3 \mathrm{~g}$ fat, $16 \mathrm{~g}$ carbs, $15 \mathrm{~g}$ sugar \\
\hline & Cherry Tomato & Several times a week & $.3 \mathrm{~g}$ fat, $5.0 \mathrm{~g}$ carbs, $4.3 \mathrm{~g}$ sugar \\
\hline & Turnip & Seasonal & $.1 \mathrm{~g}$ fat, $8.4 \mathrm{~g}$ carbs, $4.9 \mathrm{~g}$ sugar \\
\hline \multicolumn{4}{|c|}{ Japanese macaques } \\
\hline & Carrot & Several times a week & $.3 \mathrm{~g}$ fat, $12 \mathrm{~g}$ carbs, $6.1 \mathrm{~g}$ sugar \\
\hline & Celery & Several times a week & $.2 \mathrm{~g}$ fat, $3 \mathrm{~g}$ carbs, $1.4 \mathrm{~g}$ sugar \\
\hline & Green Bean & Several times a week & $.2 \mathrm{~g}$ fat, $7.0 \mathrm{~g}$ carbs, $3.3 \mathrm{~g}$ sugar \\
\hline & Jungle Pellet* & Several times a month (with enrichment) & \\
\hline & Peanut & Several times a week, enrichment & $71.0 \mathrm{~g}$ fat, $24.0 \mathrm{~g}$ carbs, $5.8 \mathrm{~g}$ sugar \\
\hline & Toasted Oats* & Several times a month (with enrichment) & $2 \mathrm{~g}$ fat, $22.4 \mathrm{~g}$ carbs, $.8 \mathrm{~g}$ sugar \\
\hline
\end{tabular}

Note. Each food's nutritional content as derived from nutritionvalue.org *complete nutritional information for jungle pellets (Scenic Jungle Food ${ }^{\mathrm{TM}}$, Marion Zoological) can be found at https://www.marionzoological.com/bird/adult/jungle/. Information for toasted oats cereal can be found at https://www.nutritionix.com/i/hospitality/toasted-oats/54f8c9dda57fd19057029c94 


\section{Subjects}

A total of 18 primates housed at Lincoln Park Zoo, Chicago, USA, participated in our study: six western lowland gorillas (Gorilla gorilla gorilla), five chimpanzees (Pan troglodytes), and seven Japanese macaques (Macaca fuscata) (Table 2). As testing was voluntary, not all members of each social group chose to participate in testing sessions. All subjects had access to water ad libitum and were given fresh produce and commercial primate chow daily.

All ape subjects lived at the Regenstein Center for African Apes at Lincoln Park Zoo (Table 2). Four of the male gorilla subjects were housed together in an all-male group, while the remaining male and female lived in a mixed-sex group with two other adult females and three juvenile females. Three female chimpanzees and one male chimpanzee subject lived in a larger mixed-sex social group comprised of four females and two males (including the subjects), while the last female chimpanzee subject lived in a separate, smaller mixed-sex social group comprised of three females (including the subject) and two males. All four ape groups lived in expansive enclosures that included both indoor and outdoor areas. Indoor areas all consisted of mulch flooring and climbing structures and were connected to outdoor areas, which included climbing structures and hammocks. Indoor and outdoor areas were connected via floor-to-ceiling sliding glass doors that allowed natural light inside (see Ross et al., 2011, for more details).

From a group of eleven Japanese macaques, consisting of five adult females, three adult males, three juvenile females, we tested seven individuals (five females and two males, Table 2). This group was housed at Lincoln Park Zoo's Regenstein Macaque Forest, a large outdoor exhibit with adjacent indoor holding areas. The outdoor area contained natural trees and vegetation, rocks, grass and mulch, and a pool (see Cronin et al., 2018, for more details).

Table 2

Test Subjects

\begin{tabular}{|c|c|c|c|}
\hline & Subject (sex, age at start of testing) & Subject group & Testing location* \\
\hline \multicolumn{4}{|l|}{ Chimpanzees } \\
\hline & Cashew $(\mathrm{F}, 24)$ & Mixed-sex & On exhibit \\
\hline & Chuckie $(\mathrm{F}, 19)$ & Mixed-sex & On exhibit \\
\hline & Kathy $(\mathrm{F}, 28)$ & Mixed-sex & On exhibit \\
\hline & Magadi (F, 27) & Mixed-sex & Off exhibit \\
\hline & Optimus (M, 19) & Mixed-sex & On exhibit \\
\hline \multicolumn{4}{|l|}{ Gorillas } \\
\hline & Amare $(\mathrm{M}, 6)$ & All male & Off exhibit \\
\hline & Azizi (M, 12) & All male & Off exhibit \\
\hline & Kwan $(\mathrm{M}, 28)$ & Mixed-sex & On exhibit \\
\hline & $\operatorname{Mosi}(\mathrm{M}, 10)$ & All male & Off exhibit \\
\hline & Rollie (F, 23) & Mixed-sex & On/off exhibit \\
\hline & Umande (M, 10) & All male & Off exhibit \\
\hline \multicolumn{4}{|c|}{ Japanese macaques } \\
\hline & Akita $(\mathrm{M}, 13)$ & Mixed-sex & On exhibit \\
\hline & Iwaki $(\mathrm{F}, 2)$ & Mixed-sex & On exhibit \\
\hline & Izumi $(\mathrm{F}, 13)$ & Mixed-sex & On exhibit \\
\hline & Mito $(\mathrm{F}, 13)$ & Mixed-sex & On exhibit \\
\hline & Miyagi (M, 13) & Mixed-sex & On exhibit \\
\hline & Nagoya $(F, 2)$ & Mixed-sex & On exhibit \\
\hline & Otaru $(\mathrm{F}, 2)$ & Mixed-sex & On exhibit \\
\hline
\end{tabular}

Note. * "on exhibit" also means that the test was conducted in public view. 


\section{Testing Environment and Apparatus}

We tested subjects via 10-point capacitive $55 \mathrm{~cm}$ ViewSonic LCD touchscreen monitors (1920 x 1080 resolution) using Zenrichment ApeTouch software (Martin, 2017). We ran testing sessions with one subject working per screen. We tested all subjects in their home enclosure, either in their main exhibit in public view or in an adjacent off-exhibit holding area, where they could stay within visual, olfactory, and auditory range of the rest of their group. Since test participation was voluntary, subjects could terminate their session at any time. Because of this, session lengths were variable and participants did not always complete the maximum number of trials per day (sensu Egelkamp et al., 2019; Huskisson et al., 2020).

We tested the chimpanzees and gorillas on a touchscreen monitor that was mounted on a mobile and adjustable cart. We began each subject's test session by placing the touchscreen flush against their enclosure mesh and verbally inviting them to participate. The subject initiated their session when they touched the screen. We terminated a session when a subject did not approach the touchscreen within five minutes, stopped participating for five minutes, or finished the maximum-allowed number of trials (30 trials/subject/day). If the subject was interrupted by another group member, we paused the session, and gave them another chance to participate once the "interrupter" moved away. We tested five of the ape subjects (four gorillas, one chimpanzee) in an off-exhibit area where they were moved temporarily each morning as part of their regular husbandry routine in order to participate in training interactions with their keepers and facilitate exhibit cleaning. The remaining subjects were tested on exhibit and in view of the public (sensu Egelkamp et al., 2019; Huskisson et al., 2020).

We tested the seven Japanese macaques in two touchscreen testing booths that were connected directly to their outdoor exhibit, which were also in view of zoo guests. Macaques could access either of the adjacent booths via a swinging door that remained unlocked while researchers were present for approximately 60 minutes of testing. Each booth measured $216 \mathrm{~cm}$ x $114 \mathrm{~cm} \times 122 \mathrm{~cm}$ and housed a touchscreen monitor. A glass panel divided the two adjacent booths, which allowed the macaques to see between the two (Cronin et al., 2018). We began a testing session with the macaques by unlocking the doors to the booths and calling out to the macaques to offer an audio cue if they did not immediately approach. We initiated a session when a macaque entered a booth. We paused a session if another macaque entered the same booth or if the subject left the booth. Two macaques could participate simultaneously in adjacent booths, and we resumed a session when a macaque re-entered if they had not yet completed the maximum number of trials for that day (50 trials/subject/day). While we aimed for each macaque to complete 30 trials per day like the apes, their daily trial maximum was set higher to allow for "makeup" trials to be collected if subjects did not complete many trials the previous test session (as their participation was completely voluntary), although this was rarely necessary. The session was terminated if a subject completed the maximum number of trials allowed for the day. Since testing was voluntary, subjects finished a variable number of trials each day, and we refer to all trials completed by each subject within a day as a "session" below.

\section{Stimuli}

For testing, we used photographs of six foods per species (apes: carrot, cucumber, grape, turnip, apple, and cherry tomato; Japanese macaques: carrot, celery, peanut, jungle pellet (Scenic ${ }^{\mathrm{TM}}$ Jungle Food, Marion Zoological), green bean, and toasted oats cereal (Gordon Food Service)), with a single photograph per food item used throughout testing (Figure 1). To create the stimuli, we photographed the food items according to Hopper et al. (2019). Additionally, for our second phase, we created a "chance" stimulus, which was a clip art image of a black question mark (hereafter referred to as the "chance symbol") (Figure 1). The background color of this stimulus matched that of the food stimuli (i.e., light gray). All subjects were familiar with the food photograph stimuli used in these tasks (Huskisson et al., 2020), but not the chance symbol, which was novel to all subjects at the beginning of this study. 
Figure 1

Food and Chance Symbol Stimuli
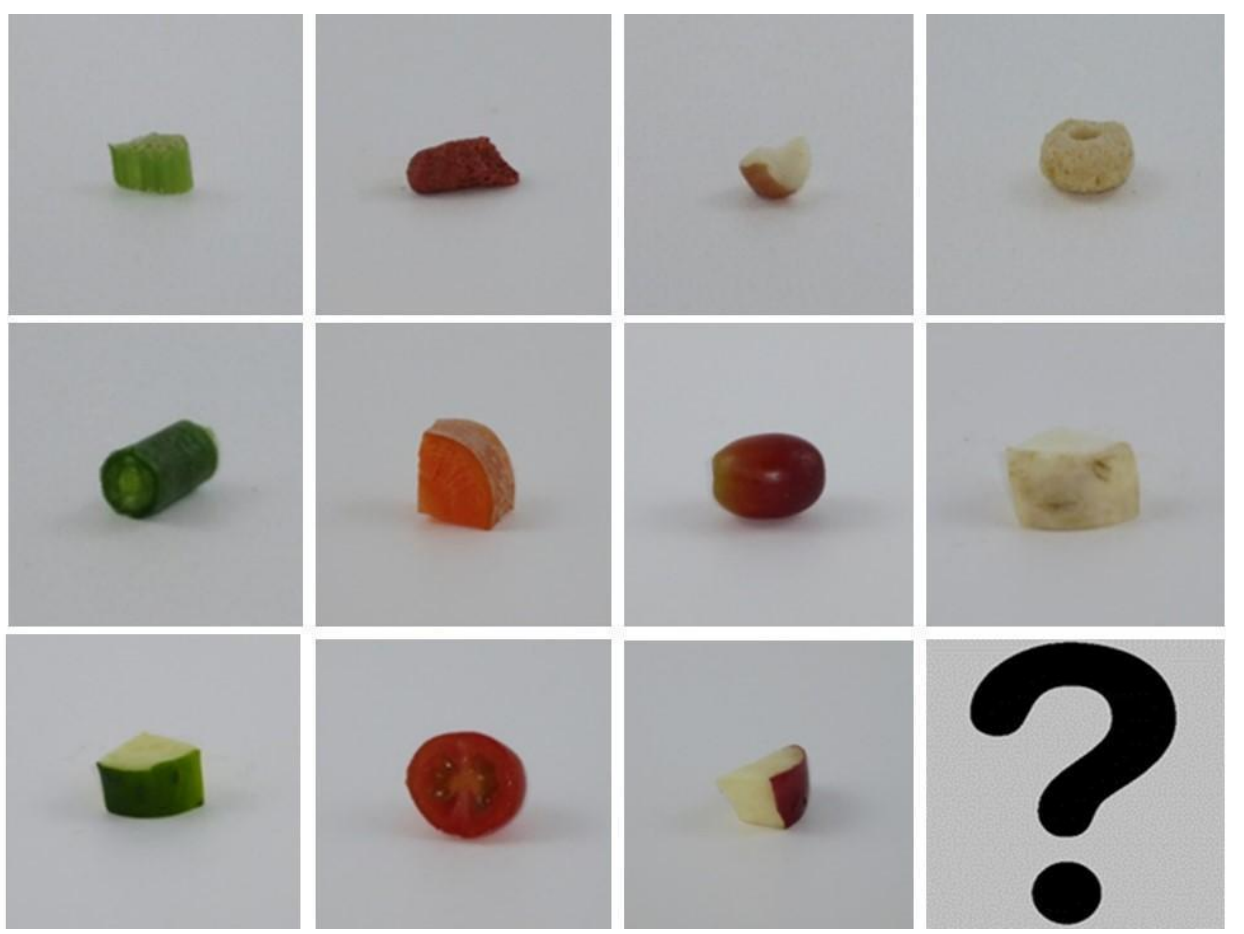

Note. From top left: celery $\uparrow$, jungle pellet (Scenic ${ }^{\mathrm{TM}}$ Jungle Food, Marion Zoological) $\uparrow$, peanut $\uparrow$, toasted oats cereal (Gordon Food Service) $\dagger$, green bean $\dagger$, carrot $\dagger$, grape*, turnip*, cucumber*, tomato*, apple*, chance symbol $\$$. *denotes ape stimulus, $\uparrow$ denotes macaque stimulus, $\$$ denotes stimulus used for all three species.

\section{Phase 1}

We tested the primates' food preferences following a forced-choice paradigm presenting two food photographs on the screen in each trial. However, the pair of photographs that we presented differed with each trial. In each test session, we randomly presented each subject with trials for each of the 15 possible pairings of the six foods. To help mitigate potential side biases in subjects' selections, the stimuli appeared in random locations across the screen that differed in each trial: there were 105 possible configurations in which the two stimuli could appear from a total of 15 locations on the screen (three rows by five columns). Each testing session consisted of no more than 30 trials for ape subjects and 50 trials for macaques. Macaques received over 30 trials on some days to make up for days in which they completed fewer trials (Average: 27.92 trials per subject per day, $S D=2.74$ ). In a trial, after subjects made a selection by pressing one of the two photographs presented on the touchscreen, we rewarded them with the food item they touched on screen. We did not use secondary reinforcers when rewarding subjects. We continued testing until each subject had completed a minimum of 25 trials per food pairing (i.e., at least 375 total trials). We tested the macaques with an 8-s inter-trial interval (ITI), to facilitate the researcher working with two monkeys simultaneously and to encourage subjects to eat rewards rather than storing them in cheek pouches, and we tested the apes with a 4-s ITI.

\section{Phase 2}

After each subject had completed all the trials for Phase 1, we tested them in Phase 2 in which we increased the level of uncertainty the primates faced when making their selections for the six foods. In each trial we presented the subjects with one of the six food photographs paired with the chance symbol (Figure 
1). In a given trial, if the subject selected the food image, we rewarded them with that food, however if they selected the chance symbol, we rewarded them with one of the six foods, selected from a predetermined randomized list (i.e., the chance symbol presented a $1 / 6$ probability of receiving each food, independent of the previous trial). Thus, in every trial, each subject had a choice between a known food option and an unknown food option (example footage of a Japanese macaque completing these trials can be viewed here: https://doi.org/10.6084/m9.figshare.10299656.v1). As for Phase 1, the two stimuli (the food photograph and the chance symbol) appeared at different random locations across the screen with each trial.

Each testing session consisted of no more than 30 trials for ape subjects and 50 trials for macaques (as for Phase 1, macaques received over 30 trials on some days to make up for days in which they completed fewer trials; Average: 27.16 trials per subject per day, $S D=2.63$ ). As in Phase 1, we rewarded subjects with their selection after touching one of the two stimuli on the screen, but they did not receive a secondary reinforcer for any selection. We required all subjects to complete a minimum of 75 trials for each of the six pairings (i.e., the six known foods each paired with the chance symbol), for a total of 450 trials. However, one gorilla, Azizi, mistakenly received 76 trials in the apple-chance condition and 74 trials in the carrotchance condition, and one macaque, Iwaki, received 74 trials in the toasted oats-chance condition. All subject ITIs remained the same as in Phase 1 (i.e., 4-s ITI for the apes and 8-s ITI for the macaques); however, the first chimpanzee tested in this phase (Optimus) received a longer 8-s ITI to facilitate his participation.

\section{Analyses}

\section{Phase 1: The Primates' Preferences and the Influence of Testing Protocol}

To determine each subject's preferences for the six foods, we analyzed their first 25 trials of each of the 15 food pairings presented in Phase 1. Specifically, we used the 'prefmod' package (Hatzinger, 2015) in R version 3.5.2 (R Core Team, 2018) to calculate each subject's "worth" values for the six foods, as well as the effect sizes of those preferences (presented as the odds of choosing one item over another). Following Hopper et al. (2019), we used a log-linear Bradley-Terry model (LLBT) to estimate each subject's worth values for each food item (Hatzinger \& Dittrich, 2012). A higher worth value is indicative of that subject's greater preference for that item relative to another (all worth values combined for a single subject total 1.0).

For a standard Bradley-Terry model the probability that a subject will prefer object $k$ to $j$, and vice versa, is:

$$
p\left(y_{j k}\right)=c_{j k}\left(\frac{\sqrt{\pi_{j}}}{\sqrt{\pi_{k}}}\right)^{y_{j k}}
$$

where $\pi_{j}$ and $\pi_{k}$ are worth values for each object on the preference scale, $y_{j k}$ is a response to the comparison of $j$ to $k$ that takes the value 1 if $j>k$ and the value -1 if $k>j$ and $c_{j k}$ is a normalizing constant. For objects $j$ and $k$, the LLBT model assumes that the observed number of selections for object $j$ instead of $k$ (and vice versa) follow a multinomial distribution conditional on a fixed number of trials. Thus, the expected number of selections for a given object over a paired trial is:

$$
m\left(y_{j k}\right)=n_{j k} p\left(y_{j k}\right)
$$

where $n_{j k}$ is the number of trials conducted for a given pair and $m\left(y_{j k}\right)$ is the expected number of selections (Hatzinger \& Dittrich, 2012). As a loglinear model, the linear predictor of the LLBT is then:

$$
\ln \ln m\left(y_{j k}\right)=u_{j k}+y_{j k}\left(\beta_{j}-\beta_{k}\right) .
$$

Here, $u_{j k}$ is a nuisance parameter whereas $\beta_{j}$ and $\beta_{k}$ correspond to the worth values on the preference scale such that $\ln \pi=2 \beta$ (Hatzinger \& Dittrich, 2012).

Once we had calculated the subjects' worth values for each of the six foods, we used the 'gnm' function (Turner \& Firth, 2015) to compare each subject's relative preference for the six foods allowing us 
to determine which foods they preferred significantly over others. The gnm function allows the user to specify and fit (by maximum likelihood) a broad class of generalized nonlinear models. As the prefmod package uses the multinomial-Poisson transformation (Baker, 1994) to fit the LLBT model through maximum likelihood, and following Hopper et al. (2019), we specified the family of the gnm function as Poisson (Hatzinger \& Dittrich, 2012).

Next, to answer our first question, as to whether primates' preferences are influenced by test presentation style, we compared the subjects' worth values for the six food, calculated in Phase 1, to their worth values for the same foods calculated from their choices in a previous study where we presented the food pairings in a blocked presentation of 90 trials per pairing (as reported in Huskisson et al., 2020). Thus, we could determine consistency of subjects' preferences across presentation methods. To do this, we created two models of the subjects' selection rates by food pairing, one that included presentation style (blocked or random) and one that did not and compared these models via the anova function in the prefmod package, with test set to "Chisq."

\section{Phase 2: The Primates' Preferences and their Responses to Incomplete Information}

To investigate the factors behind the primates' selection of an unknown food item (the chance symbol) over a known food option in Phase 2, we coded each trial as 1 (selected known food) or 0 (selected chance symbol). We used a binomial generalized linear mixed model (GLMM) and fit it using the Laplace approximation via the 'glmer' function in the lme4 package in $\mathrm{R}$ (Bates et al., 2015). We included subject ID as a random effect and the known food type as a fixed effect. We also conducted repeated measures correlations using the 'rmcorr' function (Bakdash \& Marusich, 2017) for all known food pairings for each species to see if the primates' likelihood of selecting the chance symbol over a known food item changed with time (i.e., with increasing trial number) as the subjects gained familiarity with the task and the novel chance symbol.

Next, we explored what explained subjects' differential selection of the chance symbol: 1) the strength of the subject's preference for the known food paired with the chance symbol (i.e., the worth value for the known food in a given pairing), 2) the absolute value of the food the subject received for selecting the chance symbol (i.e., the worth of the food the subject received for selecting the change symbol in a given pairing), and/or 3) the value of the food the subject received for selecting the chance symbol relative to the known food option in that same trial (i.e., the net "loss" or "gain" a subject receives from choosing the unknown option over the known food).

First, we examined whether the proportion of trials in which primates selected the chance symbol in a Phase 2 trial was negatively correlated to their preference for the known food (i.e., their worth values calculated from their responses in Phase 1) with which it was paired. Specifically, we predicted that subjects would be more likely to take a chance, and select the chance symbol, if the known food type it was paired with was a less-preferred food. We used a Spearman's rank correlation in R to test whether the subject's selection of the chance symbol was related to the strength of their preference for the alternative option in each trial - the known food.

Next, for trials in which the subject did select the chance symbol, we wished to assess if the subjects' likelihood of selecting the chance symbol again in a consecutive trial was based on the value of the reward they received for selecting the chance symbol in the preceding trial, either in absolute terms (i.e., does getting a preferred reward when selecting the chance symbol make subjects more likely to select it again?) or in relative terms (i.e., does getting a food for selecting the chance symbol that is more preferred than the known food option with which the chance symbol was paired make subjects more likely to select chance again?). To explore these two options first we coded each trial in which a subject selected the chance symbol as 1 (subject selected chance in next trial) or 0 (subject did not select chance in next trial). Additionally, for each subject, we classified the six foods as either preferred or not preferred, for which each subject's preferred foods were the three with the highest worth values and the not preferred foods were the three with the lowest worth values, as calculated from their choices in Phase 1. Thus, in a trial in which a subject selected the chance symbol, the food that they then received could be either preferred or not preferred. Similarly, the known food that the chance symbol was paired with in a given trial could be either preferred or not preferred. 
Using each subject's food value categories (preferred and not preferred), we coded the outcome of each trial in which the subject selected the chance symbol according to the value of the food the subject received, either in absolute terms or and relative to the known food option that had been paired with the chance symbol.

To test the impact of the absolute value of the reward received for selecting the chance symbol we coded each trial in which the subject received a preferred food for selecting the chance symbol as 1 , and any trial in which they received a not preferred food for selecting the chance symbol as 0 . Thus, this just considered the subject's preference for that food on their likelihood to select the chance symbol in a subsequent trial, irrespective of what other options were available to them in a given trial. Conversely, to test the impact of the value of the reward received for selecting the chance symbol relative to the value of the known food the chance symbol was paired with in that trial we coded each trial based on the food the subject received for selecting the chance symbol as either 1 (the food received was preferred, while the known food option was not preferred) or 0 (the food they received was of equivalent or less preferred value than the known food). For example, a hypothetical subject may have preferred foods that were grape, tomato, and apple and not preferred foods that were carrot, turnip, and celery. If this subject selected the chance symbol over the known choice of turnip (not preferred) and received tomato (preferred), then the trial would be classed as 1 for both absolute and relative value tests. However, if the same subject received tomato (preferred) for selecting chance, but the known option had been grape (preferred), then this trial would have been scored as 1 for the absolute value test (the food they received was preferred), but 0 for the relative value test (the food they received was not of greater value than the alternative option even though it was preferred).

For both these analyses, and as our outcome variables were binary for both models, we used binomial GLMMs to determine if subjects were more likely to select the chance symbol again in the next trial if the reward they received for selecting the chance symbol in the preceding trial was preferred in either absolute terms or relative terms. For both models, we included subject ID as a random effect and the value code of the unknown item as a fixed effect. We used the 'glmer' function in the lme4 package in R. For this specific question, macaque data was not analyzed due to errors in recording food rewards as they were given to subjects within tests.

\section{Results}

\section{Phase 1: The Primates' Preferences and the Influence of Testing Protocol}

In calculating each subject's worth values for the six foods from their choices in Phase 1, we found distinct relative preferences among all individuals and across species when comparing their worth values using the gnm function in the prefmod package (see Appendix). The exception was one male gorilla (Mosi), who showed no significant difference in his selection rates of the six foods. Although the primates showed individual variation in the relative order and strength of their preferences, we also found some within-species similarities in their choices (see Appendix). Specifically, all the macaques selected peanut at the highest rate while the gorillas' universally-preferred foods were grape and tomato. The chimpanzees showed the greatest individual variation in their most preferred food, but the three most -preferred foods were apple, tomato, and grape.

We compared subjects' worth values for the six foods calculated from their choices in Phase 1 to the worth values of those same foods when presented via blocks of 90 trials per pairing (calculated in previous testing: Huskisson et al., 2020). The majority of subjects (four gorillas, three chimpanzees, and four macaques) did not exhibit a significant difference in their worth values for the six foods across the presentation styles (one chimpanzee, Chuckie, was not included in these analyses because she did not receive one of the pairings in the previous study; Table 3). Whereas $64.70 \%$ of the subjects analyzed showed consistency in their worth values across preference test presentation styles, the remaining six subjects' (gorillas Azizi and Kwan, chimpanzee Cashew, and macaques Akita, Iwaki, and Miyagi) worth values were significantly different across the two presentation styles (blocked versus randomized; Table 3). 
Table 3

Comparisons of subjects' worth values for the six foods when presented in a blocked manner of 90 trials per pairing (data previously published in Huskisson et al., 2020) to worth values calculated from the subjects' selection rates in Phase 1 of the present study in which we presented all pairings in a randomized manner across trials.

\begin{tabular}{|c|c|c|c|}
\hline & Subject & $\mathrm{X}^{2}$ & $p$ value \\
\hline \multicolumn{4}{|l|}{ Chimpanzees } \\
\hline & Cashew $(F, 24)$ & 14.99 & .01 \\
\hline & Kathy $(\mathrm{F}, 28)$ & 1.33 & .93 \\
\hline & Magadi (F, 27) & 5.43 & .37 \\
\hline & Optimus (M, 19) & 7.20 & .21 \\
\hline \multicolumn{4}{|l|}{ Gorillas } \\
\hline & Amare $(\mathrm{M}, 6)$ & 8.43 & .13 \\
\hline & $\operatorname{Azizi}(M, 12)$ & 27.88 & $<.001$ \\
\hline & $\operatorname{Kwan}(M, 28)$ & 21.92 & $<.001$ \\
\hline & Mosi (M, 10) & 1.18 & .95 \\
\hline & Rollie (F, 23) & 5.90 & .32 \\
\hline & Umande (M, 10) & 6.68 & .25 \\
\hline \multicolumn{4}{|c|}{ Japanese macaques } \\
\hline & $\operatorname{Akita}(M, 13)$ & 51.77 & $<.001$ \\
\hline & $\operatorname{Iwaki}(F, 2)$ & 14.01 & .02 \\
\hline & Izumi $(\mathrm{F}, 13)$ & 8.96 & .11 \\
\hline & Mito $(F, 13)$ & 9.45 & .09 \\
\hline & Miyagi $(M, 13)$ & 71.59 & $<.001$ \\
\hline & Nagoya $(F, 2)$ & 10.91 & .05 \\
\hline & Otaru $(\mathrm{F}, 2)$ & 4.63 & .46 \\
\hline
\end{tabular}

Note. Model comparisons were run using the anova function in the prefmod package (family $=$ Chisq). Six subjects showed significant differences in their preferences across presentation style (highlighted in italic text). See also Figures 2 and 3.

Considering the six subjects who showed significant differences in their preferences across presentation style we found that subjects' worth value changes reflected a change in the order of preference for certain foods and/or an increase in the strengths of preference for certain foods as opposed to a complete rearrangement of all food preferences. Further, all six subjects' least- and most-preferred food remained constant across presentation styles. Cashew exhibited many changes across all pairings, and notably her preference for carrot greatly increased (Figure 2). Azizi and Kwan also showed changes across all pairings, but unlike Cashew, Azizi's preference for carrot decreased and Kwan's preference for grape increased (Figure 2). Among the macaques, Miyagi and Akita exhibited more pronounced preferences in the random presentation compared to the blocked presentation. Akita's preference for peanut increased while Miyagi and Iwaki's preference for green bean decreased (Figure 3).

\section{Phase 2: The Primates' Preferences and their Responses to Incomplete Information}

We explored all the subjects' responses in Phase 2 in which the subjects had to choose between the chance symbol and a known food item and found that all subjects selected the chance symbol across all food pairing conditions at least once. Across food pairing conditions, the average proportion of trials in which chimpanzees selected the chance option was $.44(S D=0.13), .37$ in gorillas $(S D=0.16)$, and .31 in macaques $(S D=0.12)$. We now consider each species' specific choices in turn. 
Huskisson et al. 79

Figure 2

The Three Apes, One Chimpanzee (Cashew) and Two Gorillas (Azizi and Kwan), that Showed Significant Changes in their Food Preferences Across Presentation Style

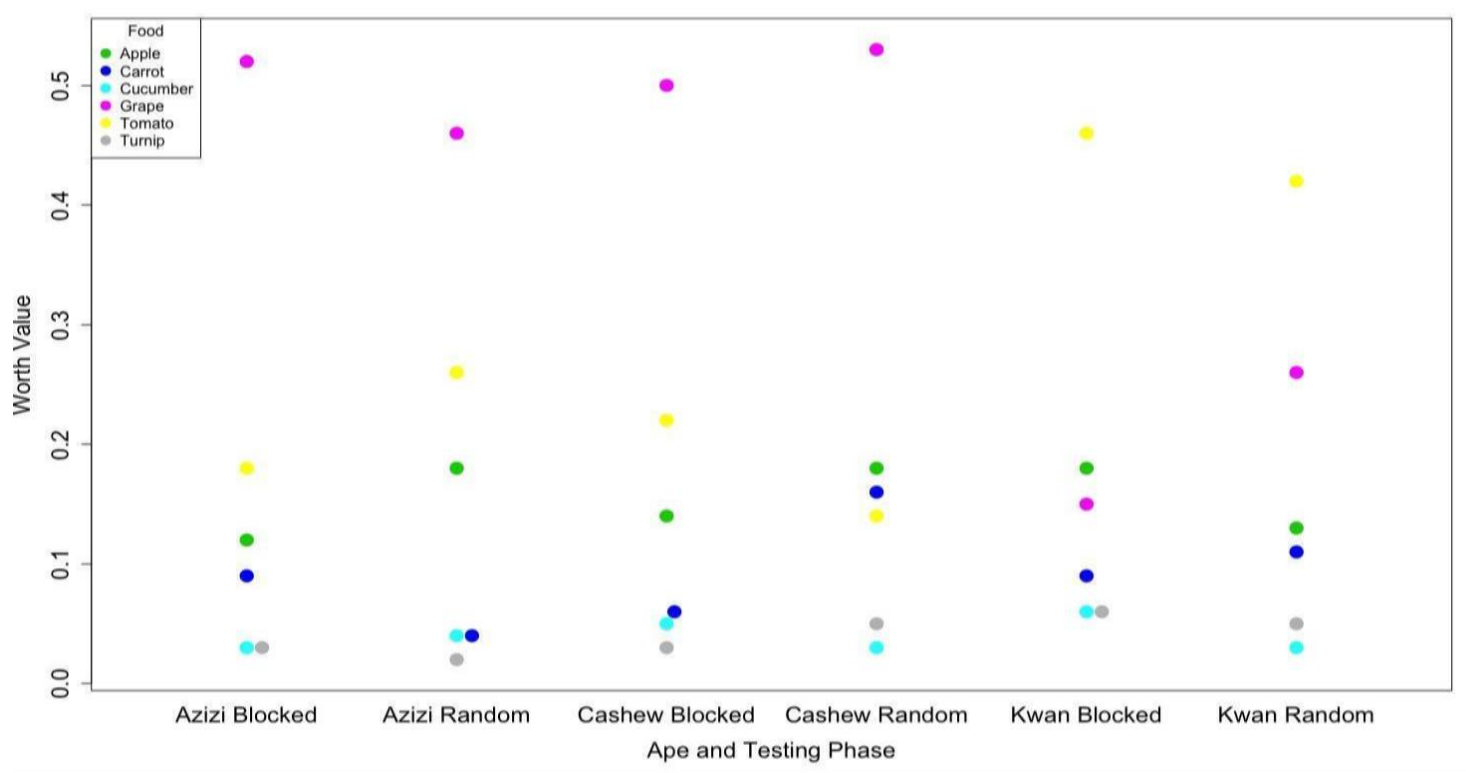

Note. Changes in preference (indicated by worth value) are shown for each food between blocked (Huskisson et al., 2020) and random (Phase 1 here) presentation styles.

\section{Figure 3}

The Three Macaques that Showed Significant Changes in their Food Preferences Across Presentation Style

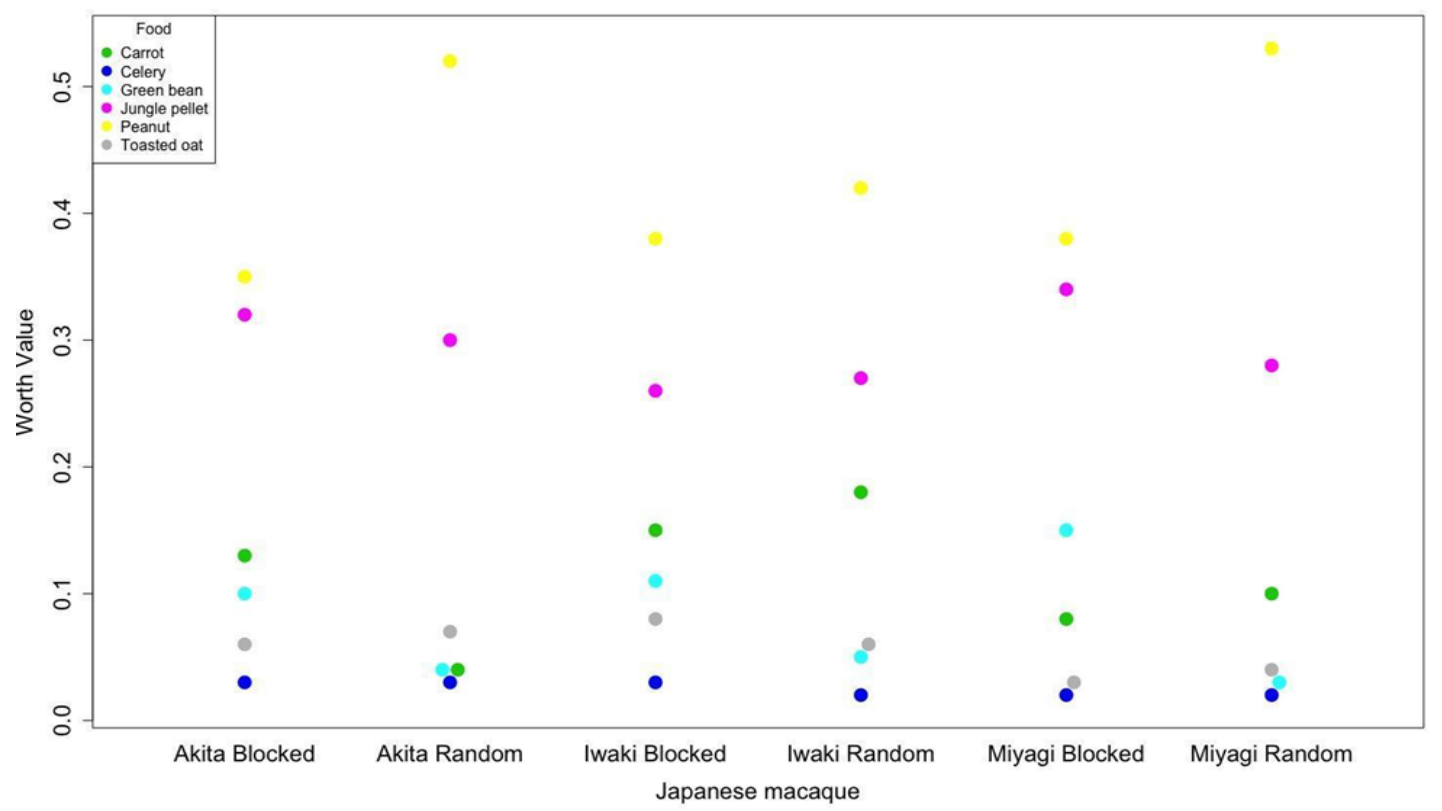

Note. Changes in preference (indicated by worth value) are shown for each food between blocked (Huskisson et al., 2020) and random (Phase 1 here) presentation styles.

Food pairing significantly impacted the chimpanzees' likelihood to select the chance symbol within a given trial, such that when the known food paired with the chance symbol was a food with a high worth 
value the chimpanzees selected that, but they selected the chance symbol more when the known food it was paired with was a less-preferred food (Figure 4 and Table 4). Specifically, chimpanzees were less likely to select the chance symbol over the known food item when the known food option was tomato. On average, the chimpanzees' worth values from Phase 1 revealed that this was a preferred food ( $M$ tomato worth value $=.19, S D=0.04)$. However, the chimpanzees were more likely to select the chance symbol when the known food alternative was cucumber $(M$ worth value $=.12, S D=0.05)$ or turnip $(M$ worth value $=.14, S D=0.06)$. Chimpanzees selected the chance symbol and known food option at equal rates when the known foods were apple $(M$ worth value $=.17, S D=0.04)$, carrot $(M$ worth value $=.15, S D=0.02)$, or grape $(M$ worth value $=.24, S D=0.17)$. Although the chimpanzees' selection of the chance symbol, when it was paired with grape as the known food option, decreased over the course of trials $(r=-.13, p=.01)$, their rate of selection of the chance symbol remained constant over trials for other food pairings (Table 5).

\section{Figure 4}

The Average Proportion of Trials in Which Chimpanzee and Gorilla Subjects Selected the Chance Symbol over the Known Food

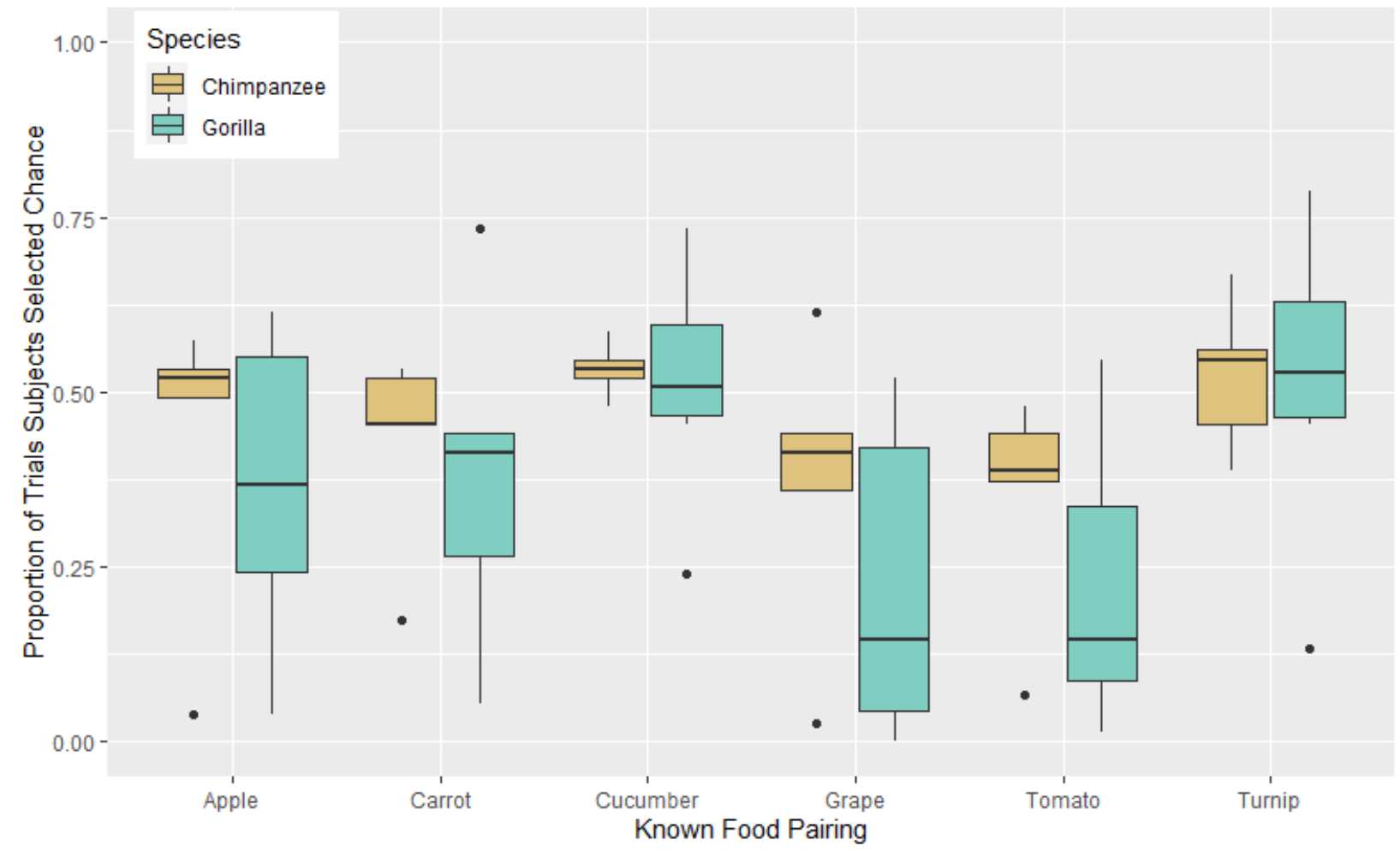

Note. Average and median proportions are shown for each food pairing presented in Phase 2. The top and bottom whiskers indicate the maximum and minimum proportion values, respectively, excluding outliers, which are represented by dots.

The degree to which gorillas selected the chance symbol over the known food item varied depending on the food that was paired with the chance symbol and, like the chimpanzees, their relative preference for the known food was related to their likelihood of selecting the chance symbol (Figure 4 and Table 4). Specifically, subjects were less likely to select the chance symbol over the known item when the known food was their preferred grape $(M$ worth value $=.29, S D=0.11)$ or tomato $(M$ worth value $=.29, S D=0.09)$, but more likely to select the chance symbol when the known item was less-preferred cucumber ( $M$ worth value $=.09, S D=0.08)$ or turnip $(M$ worth value $=0.07, S D=0.04)$. The gorillas were no more likely to select the chance symbol than the known food when the known food option was carrot $(M$ worth value $=$ $.12, S D=0.05)$ or apple $(M$ worth value $=.16, S D=0.02)$. The gorillas' selection rate of the chance symbol did not change over trials (Table 5). 
Huskisson et al. 81

Table 4

Each Species' Likelihood to Select the Chance Symbol over a Known Food Option Across Each of the Known Food Pairings

\begin{tabular}{|c|c|c|c|}
\hline Species & Known Food Pairing & $Z$ value & $p$ value \\
\hline \multicolumn{4}{|c|}{ Chimpanzee } \\
\hline & Apple & 0.15 & .88 \\
\hline & Carrot & -0.15 & .25 \\
\hline & Cucumber & 2.86 & .004 \\
\hline & Grape & -1.76 & .08 \\
\hline & Tomato & -2.38 & .02 \\
\hline & Turnip & 2.56 & .01 \\
\hline \multicolumn{4}{|l|}{ Gorilla } \\
\hline & Apple & -0.40 & .69 \\
\hline & Carrot & 0.40 & .69 \\
\hline & Cucumber & 4.63 & $<.001$ \\
\hline & Grape & -4.92 & $<.001$ \\
\hline & Tomato & -5.00 & $<.001$ \\
\hline & Turnip & 4.70 & $<.001$ \\
\hline \multicolumn{4}{|c|}{ Japanese macaque } \\
\hline & Carrot & -20.13 & $<.001$ \\
\hline & Celery & 20.12 & $<.001$ \\
\hline & Green Bean & 8.04 & $<.001$ \\
\hline & Jungle Pellet & -4.93 & $<.001$ \\
\hline & Peanut & -6.35 & $<.001$ \\
\hline & Toasted Oats & 11.45 & $<.001$ \\
\hline
\end{tabular}

Note. Positive Z-values indicate that subjects were more likely to select chance over the known food (highlighted in italic text).

In macaques, as with the apes, subjects' choices were influenced by the known food paired with the chance symbol (Figure 5, Table 4). Macaques were significantly less likely to choose the chance symbol over the known food when it was a more-preferred item like jungle pellet ( $M$ worth value $=.26, S D=0.02$ ), peanut $(M$ worth value $=.50, S D=0.04)$, or carrot $(M$ worth value $=.12, S D=.05)$. Conversely, they were more likely to select the chance symbol when it was paired with less-preferred green bean $(M$ worth value $=.05, S D=0.02)$, celery $(M$ worth value $=.02, S D=0.01)$, or toasted oats cereal $(M$ worth value $=.05, S D$ $=0.02$ ). Although the macaques' selection of the chance symbol, when it was paired with toasted oats as the known food option, increased over the course of trials $(r=.13, p=.003)$, their rate of selection of the chance symbol remained constant over trials for the other known food pairings (Table 5).

The primates' responses, as described above, suggest that their selection rate of the chance symbol was influenced by their preference for the known food with which it was paired. Therefore, our next step was to determine if the primates' preferences, as measured in Phase 1, reflected their choices in Phase 2. Only three subjects exhibited a significant positive correlation between their worth value scores for each of the foods (as calculated in Phase 1) and the proportion of trials they selected those foods when paired with the unknown option (chance symbol) in Phase 2. However, while not significant, the majority of the subjects showed a positive relationship between their food selection rates across phases (Table 6). Furthermore, one gorilla (Mosi) exhibited a non-significant negative correlation (Table 6), and we note that this was also the gorilla who did not show significant differences in the rate at which he selected the six foods in Phase 1 (Appendix). 
Huskisson et al. 82

Table 5

Repeated Measures Correlation Results for Subjects' Propensity to Select the Chance Symbol in each of the Six Known Food Pairings over Trials in Phase 2

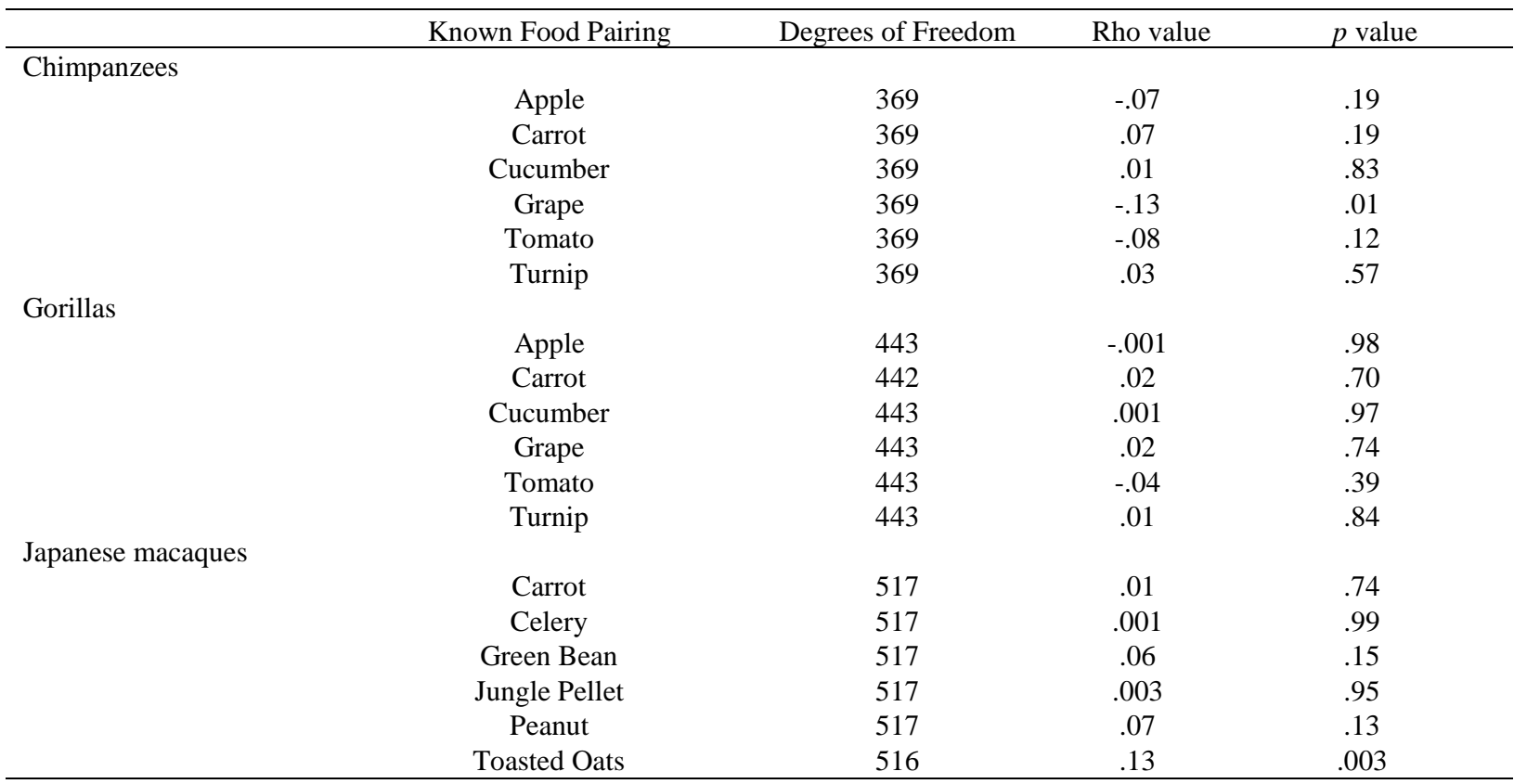

\section{Figure 5}

The Average Proportion of Trials Japanese Macaque Subjects Selected the Chance Symbol over the Known Food

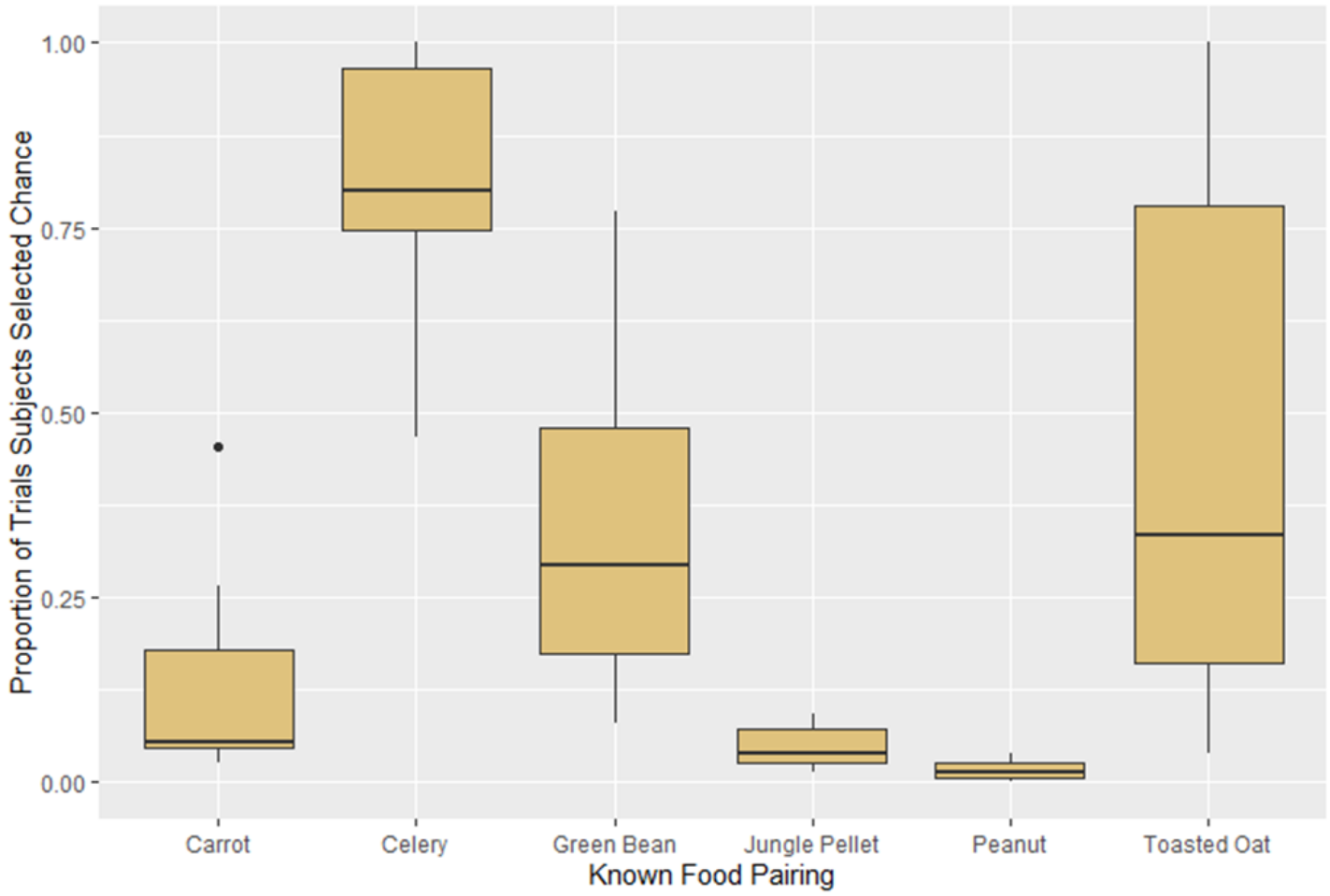

Note. Average and median proportions are shown for each food pairing presented in Phase 2. The top and bottom whiskers indicate the maximum and minimum proportion values, respectively, excluding outliers, which are represented by dots. 
Table 6

Spearman's Correlation Results for each Subject's Worth Values for the Six Foods (Phase 1) Correlated with their Rate of Selecting that Same Food over the Chance Symbol in Phase 2

\begin{tabular}{lccc}
\hline Species & Subject & Spearman's rho & $p$ value \\
\hline Chimpanzees & & .64 & .17 \\
& Cashew (F, 24) & .11 & .83 \\
Chuckie (F, 19) & .76 & .08 \\
Kathy (F, 28) & .64 & .17 \\
Magadi (F, 27) & .07 & .89 \\
Optimus (M, 19) & .91 & .01 \\
Gorillas & Amare (M, 6) & .93 & .01 \\
& Azizi (M, 12) & .72 & .11 \\
& Kwan (M, 28) & -.50 & .32 \\
Mosi (M, 10) & .49 & .33 \\
Rollie (F, 23) & .95 & .003 \\
Japanese macaques & & .79 & .06 \\
& Umande (M, 10) & .44 & .38 \\
& Akita (M, 13) & .71 & .11 \\
Iwaki (F, 2) & .66 & .16 \\
& Izumi (F, 13) & .75 & .09 \\
Mito (F, 13) & .77 & .28 \\
\hline
\end{tabular}

\section{The Primates' Preferences and their Likelihood of Taking Future Chances}

In analyzing if the absolute value of the food reward that the subjects received for selecting the chance symbol (i.e., whether it was a preferred food) influenced their likelihood to select the chance symbol again in the next trial, we found that chimpanzees were more likely to select the chance symbol in the subsequent trial if the reward received was a preferred food than if it was a not preferred food $(Z=-2.241$, $p=.03$ ). Gorillas, however, did not show variation in their likelihood to select the chance symbol in the following trial in relation to the absolute value of the food they received for selecting chance in their preceding trial $(Z=0.99, p=.32)$. Considering the relative value of the food that subjects received for selecting the chance symbol in comparison to the value of the known food paired with it, we found that chimpanzees were more likely to select the chance symbol again after receiving a reward of higher-value relative to the known food in the preceding trial in which they selected chance $(Z=-2.48, p=.01)$. Conversely, the relative value of the food reward given when a subject selected the chance symbol, as compared to the known food, did not influence gorilla subjects' likelihood to select the chance symbol in the subsequent trial $(Z=0.12, p=.91)$.

\section{Discussion}

A number of factors, both intrinsic and extrinsic, may play a role in primate decision making. Here, we explored primates' food choices under conditions of differing uncertainty, testing three primate species: chimpanzees, gorillas, and Japanese macaques. We first adapted a previously-published protocol, in which we presented pairs of foods in blocks of 90 trials, by varying which pairings were presented across trials. All subjects except one showed preferences for some foods over others, but for one-third of our subjects, their preferences differed between blocked (Huskisson et al., 2020) to randomized (Phase 1) trial presentation styles. Thus, while this change in presentation style did not alter the choices of the majority of our subjects, for six of the primates it did. This highlights the variation between and within species in their 
food preferences and their sensitivity to methodological protocols, in this case differences in uncertainty, which may in turn influence the expression of their food preferences.

In the second phase of our experiment, we increased the uncertainty under which the subjects had to make food choices. Specifically, we introduced a novel stimulus that represented an unknown choice selecting this resulted in a random reward of one of six foods. All subjects selected the chance symbol at least once, and the chimpanzees did so more often than the gorillas or macaques (on average in $44 \%$ of trials as compared to in $37 \%$ and $31 \%$ of trials for gorillas and macaques respectively). Across species, however, we found that individuals' food preferences were the best determinant of subjects' likelihood to select the chance symbol; they were more likely to take a chance when a lesser-preferred food was the alternative option. This was true for all three species tested, counter to our prediction that chimpanzees and macaques would be more likely to take chances across options than gorillas. Indeed, for many of the subjects, there was a positive relationship, which was significant for three subjects, between their worth values for each food, calculated in Phase 1, and their likelihood to select that food when it was the known option within a trial in Phase 2. However, given that we only used six foods to perform our correlations (i.e., six worth values for each food and six corresponding rates of selecting those foods), our low sample size reduces the power of our analysis. In future iterations of this task, it may be useful to incorporate a greater number of foods so as to bolster the findings from this particular analysis.

We also found that both the absolute and relative values of the food chimpanzees received when they selected the chance symbol, as compared to the known food option the chance symbol was paired with, predicted their likelihood to select the chance symbol in the subsequent trial (similar to orangutans in Pelé et al., 2014). In either case, chimpanzees not only accounted for the value of the known food when making their initial selection, but also the potential value of the outcome of their choice if they selected chance and whether they would subsequently pick the chance symbol again (this relationship was not found for gorillas, and we were unable to test this in the macaques).

Across food pairings, each of the subjects selected the chance symbol at least once in Phase 2, even though the chance symbol was arguably less "potent" than the food images: it was not a photograph, it did not depict food, it was monochrome, and it was an unfamiliar stimulus with no prior value. Thus, the subjects were willing to take chances, at least in some circumstances, although we found that they were more likely to do so when the alternative, known option, was a less-preferred food, and that, for chimpanzees at least, they were more likely to do so when they had previously received a preferred reward for doing so. Thus, their proclivity to "take a chance" was tempered, and indeed the primates' modal response across trials was to select the food photographs. Furthermore, the risk that subjects had to take was minimal - whichever stimuli they selected (food photograph or chance symbol), they still would receive a food reward, it was just the (relative) value of that reward that was unknown when selecting the chance symbol (c.f. Leinwand et al., 2020). In this case, rather than "taking a chance" perhaps the primates' selections are better characterized as "being strategic" in an attempt to maximize their chance of getting a preferred food based on the information provided in a given trial and, for chimpanzees, the value of rewards received in the preceding trial. Indeed, primates are generally risk-averse when faced with the potential for personal gains (Lakshminarayanan et al., 2011) and in captive environments, low risk-taking might be a result of animals consistently having access to (nutritional) resources, thus reducing their need to take unnecessary chances to acquire others. Conversely, risk-taking might be higher in captive animals than their wild counterparts due to plentiful food resources. This might explain our subjects' willingness to explore the chance option in the first place, while their preferences mediated the extent to which they selected it across trials. Testing subjects' choices under increased risk (i.e., with a chance of receiving no reward) might reveal primates' risk-taking more acutely (Leinwand et al., 2020; Proctor et al., 2014).

All but one of the subjects we tested showed strong individual preferences across foods. While the apes varied across individuals in both the relative order and strength of their food preferences, it is notable that the macaques showed more intra-species consistency in their food preferences than did the apes in Phase 1 (a pattern we have seen previously in this population, see Huskisson et al. 2020, for a discussion). It may be presumed that the macaques' convergence of food preferences (i.e., all preferring peanut and all disliking celery) is due to the fact that we tested them in a social setting (to enhance welfare and validity, Cronin et al., 2017), and as a result their food choices may have been socially influenced (sensu Finestone et al., 2014; 
Hopper et al., 2011). However, while we tested the macaques in their home enclosure, they had to enter individual testing booths in which they separated themselves from the social group temporarily to complete the preference tasks. Furthermore, we tested the chimpanzees with a single touchscreen in their home enclosure, giving them easy opportunities also to observe each other's choices, and yet we saw no convergence in the chimpanzees' preferences. Thus, we rule out social factors as the (sole) explanatory variable for intra-species consistency in food preferences (see also Huskisson et al., 2020). While we cannot explore all the underlying mechanisms here, the primates' preferences are likely influenced by a number of factors including the nutritional value of the food item, the taste, and familiarity of the food (see also Verspeek \& Stevens, 2020).

We note two key limitations with our study. The first is that, as with all tests of preference, we cannot clearly differentiate between a subject's selection for one option versus their avoidance of the other option. In Phase 1, subjects were given the opportunity to select foods they liked over those they did not, and thus may have come to learn to avoid less-desirable options. This avoidance response, then, may have appeared to look like a risk-taking behavior during the second phase, when in fact the primates were not "selecting the chance symbol," but rather "avoiding a low-value food option." Second, we did not counterbalance the order of blocked (Huskisson et al., 2020) and random (Phase 1 here) trial presentations among subjects. Thus, we are unable to determine if the six primates' change in food preferences was due to presentation style or simply a change over time, although we note that two-thirds of our subjects showed consistency in their preferences across presentation styles (and also over time). Future iterations of similar tasks should take care to counterbalance blocked and random presentation of paired trials. Additionally, developing methodology to tease apart the difference between preference for a certain food versus avoidance of another should be implemented, as it may highlight the nuances among individual subjects' choices.

This study details the diversity in primate food preferences and their tendencies to select an option associated with uncertainty. While these protocols are still relatively novel, they may be of use for comparative assessment of risk-taking across species, as touchscreens have been used in many non-primates (see Egelkamp \& Ross, 2019, for a review). Given the ease with which we implemented these testing paradigms, and the number of subjects evaluated, we believe that our methodology provides a feasible means to address further questions regarding differences among species in food preference and choices under increasing uncertainty (Leinwand et al., 2020). While also learning more about food preferences for management purposes, creative presentation of preference tasks may serve to enhance individual welfare and cognitive performance in other areas.

\section{Acknowledgments}

We would like to thank the animal care staff at Lincoln Park Zoo's Regenstein Center for African Apes and Regenstein Macaque Forest for their care of the primate subjects and being supportive of our research. We also thank two anonymous reviewers for constructive feedback on an earlier version of this article. This study was funded by the Leo S. Guthman Fund, the Chauncey and Marion Deering McCormick Foundation, and, at the time of writing, L.M.H. was supported by the Women's Board of Lincoln Park Zoo. This study was also supported (in part) by a grant from The David Bohnett Foundation.

\section{References}

Bakdash, J. Z., \& Marusich, L. R. (2017). Repeated measures correlation. Frontiers in Psychology, 8, 456.

Baker, S. G. (1994). The multinomial-Poisson transformation. Journal of the Royal Statistical Society: Series D, 43 (4), 495-504.

Bates, D., Mächler, M., Bolker, B. M., \& Walker, S. C. (2015). Fitting linear mixed-effects models using lme4. Journal of Statistical Software, 67(1). https://www.jstatsoft.org/article/view/v067i01

Bonnie, K. E., Bernstein-Kurtycz, L. M., Shender, M. A., Ross, S. R., \& Hopper, L. M. (2019). Foraging in a social setting: A comparative analysis of captive gorillas and chimpanzees. Primates, 60, 125-131.

Bramlett, J. L., Perdue, B. M., Evans, T. A., \& Beran, M. J. (2012). Capuchin monkeys (Cebus apella) let lesser rewards pass them by to get better rewards. Animal Cognition, 15, 963-969. 
Brosnan, S. F., Hopper, L. M., Richey, S., Freeman, H. D., Talbot, C. F., Gosling, S. D., Lambeth, S. P., \& Schapiro, S. J. (2015). Personality influences responses to inequity and contrast in chimpanzees. Animal Behavior, 101, $75-87$.

Cronin, K. A., Bethell, E. J., Jacobson, S. L., Egelkamp, C., Hopper, L. M., \& Ross, S. R. (2018). Evaluating mood changes in response to anthropogenic noise with a response-slowing task in three species of zoo-housed primates. Animal Behavior and Cognition, 5, 209-221.

Cronin, K.A., Jacobson, S. L., Bonnie, K. E., \& Hopper, L. M. (2017). Studying primate cognition in a social setting to improve validity and welfare: A literature review highlighting successful approaches. PeerJ, 5, e3649.

De Petrillo, F., \& Rosati, A. G. (2019). Ecological rationality: Convergent decision-making in apes and capuchins. Behavioural Processes, 164, 201-213.

Egelkamp, C. E., Jacobson, S. L., Wagner, K. E., Cronin, K. A., Ross, S. R., \& Hopper, L. M. (2019). A comparison of sequential learning errors made by apes and monkeys reveal individual but not species differences in learning and memory. International Journal of Comparative Psychology, 32. https://escholarship.org/uc/item/024578f5

Egelkamp, C. L., \& Ross, S. R. (2019). A review of zoo-based cognitive research using touchscreens. Zoo Biology, 38, 220-235.

Farashahi, S., Donahue, C. H., Hayden, B. Y., Lee, D., \& Soltani, A. (2019). Flexible combination of reward information across primates. Nature Human Behavior, 3, 1215-1224.

Finestone, E., Bonnie, K. E., Hopper, L. M., Vreeman, V. M., Lonsdorf, E. V., \& Ross, S. R. (2014). The interplay between individual, social, and environmental influences on chimpanzee food choices. Behavioural Processes, 105, 71-78.

Freeman, H. D., Sullivan, J., Hopper, L. M., Talbot, C. F., Holmes, A. N., Schultz-Darken, N., Williams, L. E., \& Brosnan, S. F. (2013). Different responses to reward comparisons by three primate species. PLoS One, 8, e76297.

Gray, H., Thiele, A., \& Rowe, C. (2018). Using preferred fluids and different reward schedules to motivate rhesus macaques (Macaca mulatta) in cognitive tasks. Laboratory Animals, 53(4), 372-382.

Hatzinger, R. (2015). Package "prefmod": Utilities to fit paired comparison models for preferences. https:/CRAN.Rproject.org/package=prefmod

Hatzinger, R., \& Dittrich, R. (2012). Prefmod: An R package for modeling preferences based on paired comparisons, rankings, or ratings. Journal of Statistical Software, 48, 1-31.

Haun, D. B. M., Nawroth, C., \& Call, J. (2011). Great apes' risk-taking strategies in a decision making task. PLoS One, 6, e28801.

Heilbronner, S. R., \& Hayden, B. Y. (2015). The description-experience gap in risky choice in nonhuman primates. Psychonomic Bulletin and Review, 23, 593-600.

Heilbronner, S. R., Rosati, A. G., Stevens, J. R., Hare, B., \& Hauser, M. D. (2008). A fruit in the hand or two in the bush? Divergent risk preferences in chimpanzees and bonobos. Biology Letters, 4, 246-249.

Hohmann, G., Potts, K., N'Guessan, A., Fowler, A., Mund, R., Ganzhorn, J. U., \& Ortmann, S. (2010). Plant foods consumed by Pan: Exploring the variation of nutritional ecology across Africa. American Journal of Physical Anthropology, 141, 476-485.

Hopper, L. M., Egelkamp, C. E., Fidino, M., \& Ross, S. R. (2019). An assessment of touchscreens for testing primate food preferences and valuations. Behavior Research Methods, 51, 639-650.

Hopper, L. M., Kurtycz, L. M., Ross, S. R., \& Bonnie, K. E. (2015). Captive chimpanzee foraging in a social setting: A test of problem solving, flexibility, and spatial discounting. PeerJ, 3, e833.

Hopper, L. M., Schapiro, S. J., Lambeth, S. P., \& Brosnan, S. F. (2011). Chimpanzees' socially maintained food preferences indicate both conservatism and conformity. Animal Behaviour, 81, 1195-1202.

Huskisson, S. M., Jacobson, S. L., Egelkamp, C. E., Ross, S. R., \& Hopper, L. M. (2020). Using a touchscreen paradigm to evaluate food preferences and response to novel photographic stimuli of food in three primate species (Gorilla gorilla gorilla, Pan troglodytes, and Macaca fuscata). International Journal of Primatology, 41, 523.

Lakshminarayanan, V. R., Chen, M. K., \& Santos, L. R., (2011). The evolution of decision-making under risk: Framing effects in monkey risk preferences. Journal of Experimental Social Psychology, 47, 689-693.

Leinwand, J. G., Huskisson, S. M., Egelkamp, C. L., \& Hopper, L. M. (2020). Within- and between-species variation in the responses of three primate species to a touchscreen gambling task. Learning and Motivation, 71, 101635 .

Martin, C. F. (2017). Zenrichment ApeTouch software. 
Moscovice, L. R., Issa, M. H., Petrzelkova, K. J., Kueler, N. S., Snowdon, C. T., \& Huffman, M. A. (2007). Fruit availability, chimpanzee diet, and grouping patterns on Rubondo Island, Tanzania. American Journal of Primatology, 69, 487-502.

Parrish, A. E., French, K. A., Guild, A. S., Creamer, C. L., Rossettie, M. S., \& Beran, M. J. (2020). The density bias: Capuchin monkeys (Sapajus apella) prefer densely arranged items in a food-choice task. Journal of Comparative Psychology, 134, 232-240.

Pelé, M., Broihanne, M. H., Thierry, B., Call, J., \& Dufour, V. (2014). To bet or not to bet? Decision-making under risk in non-human primates. Journal of Risk Uncertainty, 49, 141-166.

Proctor, D., Williamson, R. A., Latzman, R. D., deWaal, F. B. M., \& Brosnan, S. F. (2014). Gambling primates: Reactions to a modified Iowa Gambling Task in humans, chimpanzees and capuchin monkeys. Animal Cognition, 17, 983-995.

R Core Team. (2018). R: A language and environment for statistical computing. Vienna, Austria: R Foundation for Statistical Computing. Retrieved from www.R-project.org

Rivière, J., Apolline, K., and Meunier, H. (2019). Choice under risk of gain in tufted capuchin monkeys (Sapajus apella): A comparison with young children (Homo sapiens) and mangabey monkeys (Cercocebus torquatus torquatus). Journal of Neuroscience, Psychology, and Economics, 12, 159-168.

Rogers, M., E., Abernethy, K., Bermejo, M., Cipolletta, C., Doran, D., Mcfarland, K., Nishihara, T., Remis, M., \& Tutin, C. E. G., (2004). Western gorilla diet: A synthesis from six sites. American Journal of Primatology, 64, 173-192.

Ross, S. R., Calcutt, S., Schapiro, S. J., \& Hau, J. (2011). Space use selectivity by chimpanzees and gorillas in an indoor-outdoor enclosure. American Journal of Primatology, 73, 197-208.

Sanchez-Amaro, A., Altinok, N., Heintz, C., \& Call, J., (2019). Disentangling great apes' decoy-effect bias in a food choice task. Animal Behavior and Cognition, 6, 213-222.

Schwartz, L. P., Silberberg, A., Casey, A. H., Paukner, A., \& Suomi, S. (2016). Scaling reward value with demand curves versus preference tests. Animal Cognition, 19, 631-641.

Tsuji, Y., Kazahari, N., Kitahara, M., \& Takatsuki, S. (2008). A more detailed seasonal division of the energy balance and the protein balance of Japanese macaques (Macaca fuscata) on Kinkazan Island, northern Japan. Primates, 49, 157-160.

Turner, H., \& Firth, D. (2015). Generalized nonlinear models. http://go.warwick.ac.uk/gnm

Verspeek, J., \& Stevens, J. M. G. (2020). Food preference and nutrient composition in captive bonobos (Pan paniscus). Primates, 61, 661-671.

Vinyard, C. J., Thompson, C. L., Doherty, A., \& Robl, N. (2016). Preference and consequences: A preliminary look at whether preference impacts oral processing in non-human primates. Journal of Human Evolution, 98, 27-35. 
Huskisson et al. 88

\section{Appendix}

Results of Pairwise Comparisons run Using the gnm Function in the Prefmod Package for each Subject's Food Preferences as Calculated from their Selection Rates in Phase 1

\begin{tabular}{|c|c|c|c|c|c|}
\hline Chimpanzees & & & & & \\
\hline \multicolumn{6}{|l|}{ Cashew $(\mathrm{F}, 24)$} \\
\hline & Cucumber (.03) & Carrot (.16) & Turnip (.05) & Apple (.18) & Tomato (.14) \\
\hline Grape (.53) & $\begin{array}{c}Z=-11.27 \\
p<.001 \\
E S=0.06\end{array}$ & $\begin{array}{c}Z=\mathbf{- 8 . 8 3} \\
p<.001 \\
\mathrm{ES}=0.30\end{array}$ & $\begin{array}{c}Z=-\mathbf{9 . 5 1} \\
p<.001 \\
\mathrm{ES}=\mathbf{0 . 0 9}\end{array}$ & $\begin{array}{c}Z=-4.57 \\
p<.01 \\
\mathrm{ES}=0.34\end{array}$ & $\begin{array}{c}Z=-\mathbf{5 . 6 5} \\
p<.001 \\
\mathrm{ES}=0.26\end{array}$ \\
\hline Cucumber (.03) & & $\begin{array}{c}Z=2.59 \\
p<0.001 \\
\mathrm{ES}=\mathbf{5 . 3 3}\end{array}$ & $\begin{array}{c}Z=1.87 \\
p=.01 \\
\mathrm{ES}=\mathbf{1 . 6 7}\end{array}$ & $\begin{array}{c}Z=6.93 \\
p<.001 \\
\mathrm{ES}=\mathbf{6 . 0 0}\end{array}$ & $\begin{array}{c}Z=5.87 \\
p<.001 \\
\mathrm{ES}=\mathbf{4 . 6 7}\end{array}$ \\
\hline Carrot (.16) & & & $\begin{array}{c}Z=-.073 \\
p=.47 \\
\mathrm{ES}=0.31\end{array}$ & $\begin{array}{c}Z=4.41 \\
p<.001 \\
\mathrm{ES}=1.13\end{array}$ & $\begin{array}{c}Z=3.32 \\
p<.001 \\
\mathrm{ES}=\mathbf{0 . 8 8}\end{array}$ \\
\hline Turnip (.05) & & & & $\begin{array}{c}Z=\mathbf{5 . 1 2} \\
p<.001 \\
\mathrm{ES}=\mathbf{3 . 6 0}\end{array}$ & $\begin{array}{c}Z=4.03 \\
p<.001 \\
\mathrm{ES}=\mathbf{2 . 8 0}\end{array}$ \\
\hline Apple (.18) & & & & & $\begin{array}{c}Z=-1.10 \\
p=.27 \\
\mathrm{ES}=0.78\end{array}$ \\
\hline \multicolumn{6}{|l|}{ Chuckie (F, 19) } \\
\hline & Cucumber (.15) & Carrot (.16) & Turnip (.21) & Apple (.13) & Tomato (.17) \\
\hline Grape (.18) & $\begin{array}{c}Z=-\mathbf{0 . 8 1} \\
p<.001 \\
\mathrm{ES}=0.83\end{array}$ & $\begin{array}{c}Z=-0.46 \\
p=.64 \\
\mathrm{ES}=0.89\end{array}$ & $\begin{array}{c}Z=0.58 \\
p=.56 \\
\mathrm{ES}=1.17\end{array}$ & $\begin{array}{c}Z=-1.50 \\
p=.13 \\
\mathrm{ES}=0.87\end{array}$ & $\begin{array}{c}Z=-0.23 \\
p=.82 \\
\mathrm{ES}=0.94\end{array}$ \\
\hline Cucumber (.15) & & $\begin{array}{c}Z=0.35 \\
p=.42 \\
\mathrm{ES}=1.07\end{array}$ & $\begin{array}{c}Z=1.39 \\
p=.73 \\
\mathrm{ES}=1.40\end{array}$ & $\begin{array}{c}Z=-0.69 \\
p=.49 \\
\mathrm{ES}=0.87\end{array}$ & $\begin{array}{c}Z=0.58 \\
p=.56 \\
\mathrm{ES}=1.13\end{array}$ \\
\hline Carrot (.16) & & & $\begin{array}{c}Z=1.04 \\
p=.30 \\
\mathrm{ES}=1.31\end{array}$ & $\begin{array}{c}Z=-1.04 \\
p=.30 \\
\mathrm{ES}=0.81\end{array}$ & $\begin{array}{c}Z=0.23 \\
p=.82 \\
\mathrm{ES}=1.06\end{array}$ \\
\hline Turnip (.21) & & & & $\begin{array}{c}Z=-\mathbf{2 . 0 8} \\
p=.04 \\
\mathrm{ES}=\mathbf{0 . 6 2}\end{array}$ & $\begin{array}{c}Z=-0.81 \\
p=.42 \\
\mathrm{ES}=0.81\end{array}$ \\
\hline Apple (.13) & & & & & $\begin{array}{c}Z=1.27 \\
p=.20 \\
\mathrm{ES}=1.31\end{array}$ \\
\hline
\end{tabular}


Huskisson et al. 89

\begin{tabular}{|c|c|c|c|c|c|}
\hline \multicolumn{6}{|l|}{ Kathy (F, 28) } \\
\hline & Cucumber (.12) & Carrot (.14) & Turnip (.15) & Apple (.14) & Tomato (.26) \\
\hline Grape (.18) & $\begin{aligned} Z & =-1.62 \\
p & =.10 \\
\mathrm{ES} & =0.67\end{aligned}$ & $\begin{aligned} Z & =-1.04 \\
p & =.30 \\
\mathrm{ES} & =0.78\end{aligned}$ & $\begin{aligned} Z & =-0.81 \\
p & =.42 \\
\mathrm{ES} & =0.83\end{aligned}$ & $\begin{aligned} Z & =-1.28 \\
p & =.20 \\
\mathrm{ES} & =0.78\end{aligned}$ & $\begin{aligned} Z & =1.61 \\
p & =.11 \\
\mathrm{ES} & =1.44\end{aligned}$ \\
\hline Cucumber (.12) & & $\begin{aligned} Z & =0.58 \\
p & =.56 \\
\mathrm{ES} & =1.17\end{aligned}$ & $\begin{array}{c}Z=0.81 \\
p=.42 \\
\mathrm{ES}=1.25\end{array}$ & $\begin{aligned} Z & =0.35 \\
p & =.73 \\
\mathrm{ES} & =1.17\end{aligned}$ & $\begin{array}{c}Z=\mathbf{3 . 2 3} \\
p=. \mathbf{0 0 1} \\
\mathbf{E S}=\mathbf{2 . 1 7}\end{array}$ \\
\hline Carrot (.14) & & & $\begin{aligned} Z & =0.23 \\
p & =.82 \\
\mathrm{ES} & =1.07\end{aligned}$ & $\begin{aligned} Z & =-0.23 \\
p & =.82 \\
\mathrm{ES} & =1.00\end{aligned}$ & $\begin{aligned} Z & =2.66 \\
p & =.01 \\
\mathrm{ES} & =1.86\end{aligned}$ \\
\hline Turnip (.15) & & & & $\begin{aligned} Z & =-0.46 \\
p & =.64 \\
\mathrm{ES} & =0.93\end{aligned}$ & $\begin{aligned} Z & =2.42 \\
p & =.02 \\
\mathrm{ES} & =1.73\end{aligned}$ \\
\hline Apple (.14) & & & & & $\begin{array}{c}Z=2.89 \\
p=.003 \\
\mathrm{ES}=1.86\end{array}$ \\
\hline \multicolumn{6}{|l|}{ Magadi (F, 27) } \\
\hline & Cucumber (.17) & Carrot (.13) & Turnip (.13) & Apple (.22) & Tomato $(.20)$ \\
\hline Grape (.15) & $\begin{aligned} Z & =0.35 \\
p & =.73 \\
\mathrm{ES} & =1.13\end{aligned}$ & $\begin{aligned} Z & =-0.58 \\
p & =.56 \\
\mathrm{ES} & =0.87\end{aligned}$ & $\begin{aligned} Z & =-0.58 \\
p & =.56 \\
\mathrm{ES} & =0.87\end{aligned}$ & $\begin{aligned} Z & =1.50 \\
p & =.13 \\
\mathrm{ES} & =1.47\end{aligned}$ & $\begin{aligned} Z & =1.04 \\
p & =.30 \\
\mathrm{ES} & =1.33\end{aligned}$ \\
\hline Cucumber (.17) & & $\begin{aligned} Z & =-0.93 \\
p & =.36 \\
\mathrm{ES} & =0.76\end{aligned}$ & $\begin{aligned} Z & =-0.93 \\
p & =.36 \\
\mathrm{ES} & =0.76\end{aligned}$ & $\begin{aligned} Z & =1.16 \\
p & =.25 \\
\mathrm{ES} & =1.29\end{aligned}$ & $\begin{array}{c}Z=-0.69 \\
p=.49 \\
\mathrm{ES}=1.18\end{array}$ \\
\hline Carrot (.13) & & & $\begin{array}{c}Z=0.00 \\
p=1.00 \\
\mathrm{ES}=1.00\end{array}$ & $\begin{array}{c}Z=2.08 \\
p=.04 \\
\mathrm{ES}=1.69\end{array}$ & $\begin{aligned} Z & =1.62 \\
p & =.11 \\
\mathrm{ES} & =1.54\end{aligned}$ \\
\hline Turnip (.13) & & & & $\begin{aligned} Z & =2.08 \\
p & =.04 \\
\mathrm{ES} & =1.69\end{aligned}$ & $\begin{aligned} Z & =1.62 \\
p & =.11 \\
\mathrm{ES} & =1.54\end{aligned}$ \\
\hline Apple (.22) & & & & & $\begin{aligned} Z & =-0.46 \\
p & =.64 \\
\mathrm{ES} & =0.91\end{aligned}$ \\
\hline
\end{tabular}


Huskisson et al. 90

\begin{tabular}{|c|c|c|c|c|c|}
\hline \multicolumn{6}{|l|}{ Optimus (M, 19) } \\
\hline & Cucumber (.12) & Carrot (.18) & Turnip (.18) & Apple (.19) & Tomato (.19) \\
\hline Grape (.14) & $\begin{array}{c}Z=-0.69 \\
p=.49 \\
\mathrm{ES}=0.86\end{array}$ & $\begin{aligned} Z & =1.04 \\
p & =.30 \\
\mathrm{ES} & =1.29\end{aligned}$ & $\begin{aligned} Z & =1.04 \\
p & =.30 \\
\mathrm{ES} & =1.29\end{aligned}$ & $\begin{aligned} Z & =1.16 \\
p & =.25 \\
\mathrm{ES} & =1.36\end{aligned}$ & $\begin{aligned} Z & =1.27 \\
p & =.20 \\
\mathrm{ES} & =1.36\end{aligned}$ \\
\hline Cucumber (.12) & & $\begin{aligned} Z & =1.73 \\
p & =.08 \\
\mathrm{ES} & =1.50\end{aligned}$ & $\begin{aligned} Z & =1.73 \\
p & =.08 \\
\mathrm{ES} & =1.50\end{aligned}$ & $\begin{aligned} Z & =-1.85 \\
p & =.06 \\
\mathrm{ES} & =1.27\end{aligned}$ & $\begin{aligned} Z & =1.96 \\
p & =.05 \\
\mathrm{ES} & =1.27\end{aligned}$ \\
\hline Carrot (.18) & & & $\begin{array}{c}Z=0.00 \\
p=1.00 \\
\mathrm{ES}=1.00\end{array}$ & $\begin{aligned} Z & =0.12 \\
p & =.91 \\
\mathrm{ES} & =1.06\end{aligned}$ & $\begin{aligned} Z & =0.23 \\
p & =.82 \\
\mathrm{ES} & =1.06\end{aligned}$ \\
\hline Turnip (.18) & & & & $\begin{array}{c}Z=-0.12 \\
p=.91 \\
\mathrm{ES}=1.06\end{array}$ & $\begin{aligned} Z & =0.23 \\
p & =.82 \\
\mathrm{ES} & =1.06\end{aligned}$ \\
\hline Apple (.19) & & & & & $\begin{aligned} Z & =0.12 \\
p & =.91 \\
\mathrm{ES} & =1.00\end{aligned}$ \\
\hline \multicolumn{6}{|l|}{ Gorillas } \\
\hline \multicolumn{6}{|l|}{ Amare $(\mathrm{M}, 6)$} \\
\hline & Cucumber (.04) & Carrot (.12) & Turnip (.03) & Apple (.15) & Tomato (.34) \\
\hline Grape (.32) & $\begin{array}{c}Z=8.76 \\
p<.001 \\
\mathrm{ES}=\mathbf{0 . 1 3}\end{array}$ & $\begin{array}{c}Z=4.22 \\
p<.001 \\
\mathrm{ES}=\mathbf{0 . 3 8}\end{array}$ & $\begin{array}{c}Z=8.76 \\
p<.001 \\
\mathrm{ES}=\mathbf{0 . 1 0}\end{array}$ & $\begin{array}{c}Z=\mathbf{3 . 2 4} \\
p=.001 \\
\mathbf{E S}=\mathbf{0 . 4 7}\end{array}$ & $\begin{array}{c}Z=-0.23 \\
p=.82 \\
\mathrm{ES}=1.06\end{array}$ \\
\hline Cucumber (.04) & & $\begin{array}{c}Z=4.68 \\
p<.001 \\
\mathrm{ES}=\mathbf{3 . 0 0}\end{array}$ & $\begin{aligned} Z & =-0.57 \\
p & =.57 \\
\mathrm{ES} & =0.75\end{aligned}$ & $\begin{array}{c}Z=5.64 \\
p<.001 \\
\text { ES }=3.75\end{array}$ & $\begin{array}{c}Z=8.98 \\
p<.001 \\
\mathrm{ES}=\mathbf{8 . 5 0}\end{array}$ \\
\hline Carrot (.12) & & & $\begin{array}{c}Z=-5.23 \\
p<.001 \\
\mathrm{ES}=0.25\end{array}$ & $\begin{aligned} Z & =0.99 \\
p & =.32 \\
\mathrm{ES} & =1.25\end{aligned}$ & $\begin{array}{c}Z=4.44 \\
p<.001 \\
\mathrm{ES}=2.83\end{array}$ \\
\hline Turnip (.03) & & & & $\begin{array}{c}Z=\mathbf{6 . 1 9} \\
p<.001 \\
\mathrm{ES}=5.00\end{array}$ & $\begin{array}{c}Z=9.51 \\
p<.001 \\
\mathrm{ES}=\mathbf{1 1 . 3 3}\end{array}$ \\
\hline Apple (.15) & & & & & $\begin{array}{c}Z=\mathbf{3 . 4 7} \\
p<.001 \\
\mathbf{E S}=\mathbf{2 . 2 7}\end{array}$ \\
\hline
\end{tabular}


Huskisson et al. 91

\begin{tabular}{|c|c|c|c|c|c|}
\hline \multicolumn{6}{|l|}{ Azizi (M, 12) } \\
\hline & Cucumber (.04) & Carrot (.04) & Turnip (.02) & Apple (.18) & Tomato (.26) \\
\hline Grape (.46) & $\begin{array}{c}Z=-\mathbf{9 . 5 5} \\
p<.001 \\
\mathrm{ES}=0.09\end{array}$ & $\begin{array}{c}Z=-\mathbf{9 . 8 9} \\
p<.001 \\
\mathbf{E S}=\mathbf{0 . 0 9}\end{array}$ & $\begin{array}{c}\boldsymbol{Z}=\mathbf{- \mathbf { 1 2 . 1 4 }} \\
\boldsymbol{p}<\mathbf{. 0 0 1} \\
\mathrm{ES}=0.04\end{array}$ & $\begin{array}{c}Z=\mathbf{- 3 . 8 8} \\
p<.001 \\
\mathbf{E S}=\mathbf{0 . 3 9}\end{array}$ & $\begin{aligned} Z & =\mathbf{- 2 . 4 3} \\
p & =\mathbf{. 0 2} \\
\mathrm{ES} & =0.57\end{aligned}$ \\
\hline Cucumber (.04) & & $\begin{array}{c}Z=-0.37 \\
p=.71 \\
\mathrm{ES}=1.00\end{array}$ & $\begin{array}{c}Z=-\mathbf{2 . 8 2} \\
p=.005 \\
\mathbf{E S}=\mathbf{0 . 5 0}\end{array}$ & $\begin{array}{c}Z=5.87 \\
p<.001 \\
\mathbf{E S}=\mathbf{4 . 5 0}\end{array}$ & $\begin{array}{c}Z=7.28 \\
p<.001 \\
\mathbf{E S}=\mathbf{6 . 5 0}\end{array}$ \\
\hline Carrot (.04) & & & $\begin{array}{c}Z=\mathbf{- 2 . 4 5} \\
p=.01 \\
\mathrm{ES}=0.50\end{array}$ & $\begin{array}{c}Z=6.23 \\
p<.001 \\
\mathrm{ES}=4.50\end{array}$ & $\begin{array}{c}Z=7.63 \\
p<.001 \\
\mathrm{ES}=\mathbf{6 . 5 0}\end{array}$ \\
\hline Turnip (.02) & & & & $\begin{array}{c}Z=\mathbf{8 . 5 5} \\
p<.001 \\
\mathbf{E S}=\mathbf{9 . 0 0}\end{array}$ & $\begin{array}{c}Z=9.92 \\
p<.001 \\
\mathrm{ES}=\mathbf{1 3 . 0 0}\end{array}$ \\
\hline Apple (.18) & & & & & $\begin{array}{c}Z=1.47 \\
p=.14 \\
\mathrm{ES}=1.44\end{array}$ \\
\hline \multicolumn{6}{|l|}{ Kwan (M, 28) } \\
\hline Grape (.26) & $\begin{array}{c}Z=-\mathbf{8 . 2 5} \\
p<.001 \\
\mathrm{ES}=\mathbf{0 . 1 2}\end{array}$ & $\begin{array}{c}Z=-\mathbf{3 . 2 7} \\
p=.001 \\
\mathrm{ES}=\mathbf{0 . 4 2}\end{array}$ & $\begin{array}{c}Z=-6.82 \\
p<.001 \\
\mathrm{ES}=0.19\end{array}$ & $\begin{array}{c}Z=-2.91 \\
p=.004 \\
\mathrm{ES}=0.50\end{array}$ & $\begin{aligned} Z & =2.19 \\
p & =.03 \\
\mathrm{ES} & =1.62\end{aligned}$ \\
\hline Cucumber (.03) & & $\begin{array}{c}Z=5.08 \\
p<.001 \\
\mathrm{ES}=\mathbf{3 . 6 7}\end{array}$ & $\begin{aligned} Z & =1.49 \\
p & =.14 \\
\mathrm{ES} & =1.67\end{aligned}$ & $\begin{array}{c}Z=5.44 \\
p<.001 \\
\mathrm{ES}=\mathbf{4 . 3 3}\end{array}$ & $\begin{array}{c}Z=10.31 \\
p<.001 \\
\mathrm{ES}=14.00\end{array}$ \\
\hline Carrot (.11) & & & $\begin{array}{c}Z=-3.62 \\
p<.001 \\
\mathrm{ES}=\mathbf{0 . 4 5}\end{array}$ & $\begin{aligned} Z & =0.37 \\
p & =.71 \\
\mathrm{ES} & =1.18\end{aligned}$ & $\begin{array}{c}Z=5.42 \\
p<.001 \\
\mathrm{ES}=\mathbf{3 . 8 2}\end{array}$ \\
\hline Turnip (.05) & & & & $\begin{array}{c}Z=\mathbf{3 . 9 8} \\
p<.001 \\
\mathrm{ES}=\mathbf{2 . 6 0}\end{array}$ & $\begin{array}{c}Z=8.91 \\
p<.001 \\
\mathrm{ES}=8.40\end{array}$ \\
\hline Apple (.13) & & & & & $\begin{array}{c}Z=5.06 \\
p<.001 \\
\mathrm{ES}=\mathbf{3 . 2 3}\end{array}$ \\
\hline
\end{tabular}


Huskisson et al. 92

\begin{tabular}{|c|c|c|c|c|c|}
\hline \multicolumn{6}{|l|}{ Mosi (M, 10) } \\
\hline & Cucumber (.17) & Carrot (.18) & Turnip (.13) & Apple (.18) & Tomato (.19) \\
\hline Grape (.16) & $\begin{array}{c}Z=0.23 \\
p=.82 \\
\mathrm{ES}=1.06\end{array}$ & $\begin{array}{c}Z=0.58 \\
p=.56 \\
\mathrm{ES}=1.13\end{array}$ & $\begin{array}{c}Z=-0.92 \\
p=.36 \\
\mathrm{ES}=0.81\end{array}$ & $\begin{array}{c}Z=0.46 \\
p=.64 \\
\mathrm{ES}=1.13\end{array}$ & $\begin{array}{c}Z=0.69 \\
p=.49 \\
\mathrm{ES}=1.19\end{array}$ \\
\hline Cucumber (.17) & & $\begin{array}{c}Z=0.35 \\
p=.73 \\
\mathrm{ES}=1.06\end{array}$ & $\begin{array}{c}Z=-1.15 \\
p=.25 \\
\mathrm{ES}=0.76\end{array}$ & $\begin{array}{c}Z=0.23 \\
p=.82 \\
\mathrm{ES}=1.06\end{array}$ & $\begin{array}{c}Z=0.46 \\
p=.64 \\
\mathrm{ES}=1.12\end{array}$ \\
\hline Carrot (.18) & & & $\begin{array}{c}Z=-1.50 \\
p=.13 \\
\mathrm{ES}=0.72\end{array}$ & $\begin{array}{c}Z=-0.12 \\
p=.91 \\
\mathrm{ES}=1.00\end{array}$ & $\begin{array}{c}Z=0.12 \\
p=.91 \\
\mathrm{ES}=1.06\end{array}$ \\
\hline Turnip (.13) & & & & $\begin{array}{c}Z=1.39 \\
p=.17 \\
\mathrm{ES}=1.38\end{array}$ & $\begin{array}{c}Z=1.62 \\
p=.11 \\
\mathrm{ES}=1.46\end{array}$ \\
\hline Apple (.18) & & & & & $\begin{array}{c}Z=0.23 \\
p=.82 \\
\mathrm{ES}=1.06\end{array}$ \\
\hline \multicolumn{6}{|l|}{ Rollie (F, 23) } \\
\hline & Cucumber (.22) & Carrot (.15) & Turnip (.11) & Apple (.15) & Tomato (.20) \\
\hline Grape (.17) & $\begin{array}{c}Z=1.04 \\
p=.30 \\
\mathrm{ES}=1.29\end{array}$ & $\begin{array}{c}Z=-0.58 \\
p=.56 \\
\mathrm{ES}=0.88\end{array}$ & $\begin{array}{c}Z=-2.08 \\
p=.56 \\
\mathrm{ES}=0.65\end{array}$ & $\begin{array}{c}Z=-0.70 \\
p=.49 \\
\mathrm{ES}=0.88\end{array}$ & $\begin{array}{c}Z=0.58 \\
p=.56 \\
\mathrm{ES}=1.18\end{array}$ \\
\hline Cucumber (.22) & & $\begin{array}{c}Z=-1.62 \\
p=.11 \\
\mathrm{ES}=0.68\end{array}$ & $\begin{array}{c}Z=\mathbf{- 3 . 1 2} \\
p=\mathbf{0 0 2} \\
\mathrm{ES}=\mathbf{0 . 5 0}\end{array}$ & $\begin{array}{c}Z=-1.74 \\
p=.08 \\
\mathrm{ES}=0.68\end{array}$ & $\begin{array}{c}Z=-0.46 \\
p=.64 \\
\mathrm{ES}=0.91\end{array}$ \\
\hline Carrot (.15) & & & $\begin{array}{c}Z=-1.50 \\
p=.13 \\
\mathrm{ES}=0.73\end{array}$ & $\begin{array}{c}Z=-0.12 \\
p=.91 \\
\mathrm{ES}=1.00\end{array}$ & $\begin{array}{c}Z=1.16 \\
p=.25 \\
\mathrm{ES}=1.33\end{array}$ \\
\hline Turnip (.11) & & & & $\begin{array}{c}Z=1.39 \\
p=.17 \\
\mathrm{ES}=1.36\end{array}$ & $\begin{array}{c}Z=2.66 \\
p=.01 \\
\mathrm{ES}=1.82\end{array}$ \\
\hline Apple (.15) & & & & & $\begin{array}{c}Z=1.28 \\
p=.20 \\
\mathrm{ES}=1.33\end{array}$ \\
\hline
\end{tabular}


Huskisson et al. 93

\begin{tabular}{|c|c|c|c|c|c|}
\hline \multicolumn{6}{|l|}{ Umande $(\mathrm{M}, 10)$} \\
\hline & Cucumber (.06) & Carrot (.11) & Turnip (.05) & Apple (.16) & Tomato (.32) \\
\hline Grape (.35) & $\begin{array}{c}Z=-7.27 \\
p<.001 \\
\mathrm{ES}=0.17\end{array}$ & $\begin{array}{c}Z=-4.95 \\
p<.001 \\
\mathrm{ES}=0.31\end{array}$ & $\begin{array}{c}Z=-7.82 \\
p<.001 \\
\mathrm{ES}=0.14\end{array}$ & $\begin{array}{c}Z=-4.60 \\
p<.001 \\
\mathrm{ES}=0.46\end{array}$ & $\begin{aligned} Z & =-0.34 \\
p & =.73 \\
\mathrm{ES} & =0.91\end{aligned}$ \\
\hline Cucumber (.06) & & $\begin{array}{c}Z=2.38 \\
p=.02 \\
\mathrm{ES}=1.83\end{array}$ & $\begin{array}{c}Z=-0.58 \\
p=.56 \\
\mathrm{ES}=0.83\end{array}$ & $\begin{array}{c}Z=2.74 \\
p=.01 \\
\mathrm{ES}=2.67\end{array}$ & $\begin{array}{c}Z=6.93 \\
p<.001 \\
\mathbf{E S}=\mathbf{5 . 3 3}\end{array}$ \\
\hline Carrot (.11) & & & $\begin{array}{c}Z=-2.95 \\
p=.003 \\
\mathrm{ES}=\mathbf{0 . 4 5}\end{array}$ & $\begin{aligned} Z & =0.36 \\
p & =.72 \\
\mathrm{ES} & =1.45\end{aligned}$ & $\begin{array}{c}Z=4.62 \\
p<.001 \\
\mathrm{ES}=\mathbf{2 . 9 1}\end{array}$ \\
\hline Turnip (.05) & & & & $\begin{array}{c}Z=3.31 \\
p<.001 \\
\mathrm{ES}=\mathbf{3 . 2 0}\end{array}$ & $\begin{array}{c}Z=7.49 \\
p<.001 \\
\mathrm{ES}=6.40\end{array}$ \\
\hline Apple (.16) & & & & & $\begin{array}{c}Z=4.26 \\
p<.001 \\
\mathrm{ES}=\mathbf{2 . 0 0}\end{array}$ \\
\hline \multicolumn{6}{|l|}{ Japanese macaques } \\
\hline Akita $(\mathrm{M}, 13)$ & Jungle Pellet (.30) & Carrot (.04) & Celery (.03) & Toasted Oat (.07) & Green Bean (.04) \\
\hline Peanut (.52) & $\begin{array}{c}Z=-\mathbf{2 . 3 5} \\
p=.02 \\
\mathrm{ES}=0.58\end{array}$ & $\begin{array}{c}Z=-10.11 \\
p<.001 \\
\mathrm{ES}=0.08\end{array}$ & $\begin{array}{c}Z=-11.65 \\
p<.001 \\
\mathrm{ES}=0.06\end{array}$ & $\begin{array}{c}Z=-8.01 \\
p<.001 \\
\mathrm{ES}=0.13\end{array}$ & $\begin{array}{c}Z=-\mathbf{1 0 . 3 3} \\
p<.001 \\
\mathrm{ES}=\mathbf{0 . 0 8}\end{array}$ \\
\hline Jungle Pellet (.30) & & $\begin{array}{c}Z=-7.92 \\
p<.001 \\
\text { ES }=0.13\end{array}$ & $\begin{array}{c}Z=-9.49 \\
p<.001 \\
\text { ES }=0.10\end{array}$ & $\begin{array}{c}Z=-5.77 \\
p<.001 \\
\mathrm{ES}=0.23\end{array}$ & $\begin{array}{c}Z=-\mathbf{8 . 1 5} \\
p<.001 \\
\mathrm{ES}=0.13\end{array}$ \\
\hline Carrot (.04) & & & $\begin{array}{c}Z=-1.68 \\
p=.09 \\
\mathrm{ES}=0.75\end{array}$ & $\begin{array}{c}Z=2.25 \\
p=.02 \\
\mathrm{ES}=1.75\end{array}$ & $\begin{aligned} Z & =-0.25 \\
p & =.81 \\
\mathrm{ES} & =1.00\end{aligned}$ \\
\hline Celery (.03) & & & & $\begin{array}{c}Z=\mathbf{3 . 9 1} \\
p<.001 \\
\mathrm{ES}=\mathbf{2 . 3 3}\end{array}$ & $\begin{array}{c}Z=-1.43 \\
p=.15 \\
\mathrm{ES}=1.33\end{array}$ \\
\hline Toasted Oat (.07) & & & & & $\begin{array}{c}Z=-\mathbf{2 . 4 9} \\
p=.01 \\
\mathrm{ES}=\mathbf{0 . 5 7}\end{array}$ \\
\hline
\end{tabular}


Huskisson et al. 94

\begin{tabular}{|c|c|c|c|c|c|}
\hline \multicolumn{6}{|l|}{ Iwaki $(\mathrm{F}, 2)$} \\
\hline & Jungle Pellet (.27) & Carrot (.18) & Celery (.02) & Toasted Oat (.06) & Green Bean (.05) \\
\hline Peanut (.42) & $\begin{aligned} Z & =-1.97 \\
p & =.05 \\
\mathrm{ES} & =0.64\end{aligned}$ & $\begin{array}{c}Z=-\mathbf{3 . 5 4} \\
p<.001 \\
\mathrm{ES}=\mathbf{0 . 4 3}\end{array}$ & $\begin{array}{c}Z=\mathbf{- 1 1 . 9 2} \\
\boldsymbol{p}<\mathbf{. 0 0 1} \\
\mathrm{ES}=0.05\end{array}$ & $\begin{array}{c}Z=\mathbf{- 7 . 7 8} \\
\boldsymbol{p}<. \mathbf{0 0 1} \\
\mathrm{ES}=0.14\end{array}$ & $\begin{array}{c}Z=-\mathbf{8 . 3 6} \\
p<.001 \\
\mathrm{ES}=\mathbf{0 . 1 2}\end{array}$ \\
\hline Jungle Pellet (.27) & & $\begin{array}{c}Z=-1.58 \\
p=.11 \\
\mathrm{ES}=0.67\end{array}$ & $\begin{array}{c}Z=-10.09 \\
p<.001 \\
\mathrm{ES}=0.07\end{array}$ & $\begin{array}{c}Z=-5.89 \\
p<.001 \\
\mathrm{ES}=0.22\end{array}$ & $\begin{array}{c}Z=-6.50 \\
p<.001 \\
\mathrm{ES}=0.19\end{array}$ \\
\hline Carrot (.18) & & & $\begin{array}{c}Z=\mathbf{- 8 . 6 1} \\
p<.001 \\
\mathrm{ES}=0.11\end{array}$ & $\begin{array}{c}Z=-4.35 \\
p<.001 \\
\mathrm{ES}=\mathbf{0 . 3 3}\end{array}$ & $\begin{array}{c}Z=-4.95 \\
p<.001 \\
\mathrm{ES}=0.28\end{array}$ \\
\hline Celery (.02) & & & & $\begin{array}{c}Z=4.41 \\
p<.001 \\
\mathrm{ES}=\mathbf{3 . 0 0}\end{array}$ & $\begin{array}{c}Z=\mathbf{3 . 8 1} \\
p<.001 \\
\mathrm{ES}=\mathbf{2 . 5 0}\end{array}$ \\
\hline Toasted Oat (.06) & & & & & $\begin{aligned} Z & =-0.62 \\
p & =.54 \\
\mathrm{ES} & =0.83\end{aligned}$ \\
\hline \multicolumn{6}{|l|}{ Izumi $(\mathrm{F}, 13)$} \\
\hline Peanut (.53) & $\begin{array}{c}Z=-\mathbf{3 . 3 0} \\
p<.001 \\
\mathrm{ES}=\mathbf{0 . 4 7}\end{array}$ & $\begin{array}{c}Z=-6.83 \\
p<.001 \\
\mathrm{ES}=0.19\end{array}$ & $\begin{array}{c}Z=-13.19 \\
p<.001 \\
\text { ES }=0.04\end{array}$ & $\begin{array}{c}Z=-11.73 \\
p<.001 \\
\mathrm{ES}=0.06\end{array}$ & $\begin{array}{c}Z=-\mathbf{8 . 4 1} \\
p<.001 \\
\mathrm{ES}=\mathbf{0 . 1 3}\end{array}$ \\
\hline Jungle Pellet (.25) & & $\begin{array}{c}Z=-3.63 \\
p<.001 \\
\mathrm{ES}=0.40\end{array}$ & $\begin{array}{c}Z=-10.17 \\
p<.001 \\
\mathrm{ES}=0.08\end{array}$ & $\begin{array}{c}Z=-8.68 \\
p<.001 \\
\mathrm{ES}=0.12\end{array}$ & $\begin{array}{c}Z=-4.89 \\
p<.001 \\
\mathrm{ES}=0.28\end{array}$ \\
\hline Carrot (.10) & & & $\begin{array}{c}Z=-6.76 \\
p<.001 \\
\mathrm{ES}=0.20\end{array}$ & $\begin{array}{c}Z=-5.21 \\
p<.001 \\
\mathrm{ES}=\mathbf{0 . 3 0}\end{array}$ & $\begin{array}{c}Z=-1.29 \\
p=.20 \\
\mathrm{ES}=0.70\end{array}$ \\
\hline Celery (.02) & & & & $\begin{aligned} Z & =1.61 \\
p & =.11 \\
\mathrm{ES} & =1.50\end{aligned}$ & $\begin{array}{c}Z=5.52 \\
p<.001 \\
\mathbf{E S}=\mathbf{3 . 5 0}\end{array}$ \\
\hline Toasted Oat (.03) & & & & & $\begin{array}{c}Z=4.00 \\
p<.001 \\
\mathrm{ES}=\mathbf{2 . 3 3}\end{array}$ \\
\hline
\end{tabular}


Huskisson et al. 95

\begin{tabular}{|c|c|c|c|c|c|}
\hline \multicolumn{6}{|l|}{ Mito $(F, 13)$} \\
\hline & Jungle Pellet (.26) & Carrot (.10) & Celery (.01) & Toasted Oat (.02) & Green Bean (.06) \\
\hline Peanut (.53) & $\begin{array}{c}Z=\mathbf{- 3 . 0 8} \\
p<.001 \\
\mathrm{ES}=\mathbf{0 . 4 9}\end{array}$ & $\begin{array}{c}Z=-6.64 \\
p<.001 \\
E S=0.19\end{array}$ & $\begin{array}{c}Z=-14.09 \\
p<.001 \\
\mathrm{ES}=0.02\end{array}$ & $\begin{array}{c}Z=-11.82 \\
p<.001 \\
\text { ES }=0.04\end{array}$ & $\begin{array}{c}Z=-8.36 \\
p<.001 \\
\text { ES }=0.11\end{array}$ \\
\hline Jungle Pellet (.26) & & $\begin{array}{c}Z=\mathbf{- 3 . 6 5} \\
p=\mathbf{0 0 2} \\
\mathrm{ES}=\mathbf{0 . 3 8}\end{array}$ & $\begin{array}{c}Z=-11.31 \\
p<.001 \\
\mathrm{ES}=0.04\end{array}$ & $\begin{array}{c}Z=-\mathbf{9 . 0 2} \\
p<.001 \\
\mathbf{E S}=\mathbf{0 . 0 8}\end{array}$ & $\begin{array}{c}Z=-5.44 \\
p<.001 \\
\mathrm{ES}=\mathbf{0 . 2 3}\end{array}$ \\
\hline Carrot (.10) & & & $\begin{array}{c}Z=-7.93 \\
p<.001 \\
\mathbf{E S}=\mathbf{0 . 1 0}\end{array}$ & $\begin{array}{c}Z=-5.55 \\
p<.001 \\
\mathbf{E S}=\mathbf{0 . 2 0}\end{array}$ & $\begin{array}{c}Z=-1.83 \\
p=.07 \\
\mathrm{ES}=0.60\end{array}$ \\
\hline Celery (.01) & & & & $\begin{array}{c}Z=\mathbf{2 . 5 2} \\
p=.01 \\
\mathrm{ES}=\mathbf{2 . 0 0}\end{array}$ & $\begin{array}{c}Z=6.21 \\
p<.001 \\
\mathrm{ES}=\mathbf{6 . 0 0}\end{array}$ \\
\hline Toasted Oat (.02) & & & & & $\begin{array}{c}Z=\mathbf{3 . 7 6} \\
p<.001 \\
\mathrm{ES}=\mathbf{3 . 0 0}\end{array}$ \\
\hline \multicolumn{6}{|l|}{ Miyagi (M, 13) } \\
\hline & Jungle Pellet (.28) & Carrot (.10) & Celery (.02) & Toasted Oat (.04) & Green Bean (.03) \\
\hline Peanut (.53) & $\begin{array}{c}Z=-\mathbf{2 . 8 2} \\
p=.004 \\
\mathrm{ES}=\mathbf{0 . 5 3}\end{array}$ & $\begin{array}{c}Z=-6.70 \\
p<.001 \\
\mathrm{ES}=0.19\end{array}$ & $\begin{array}{c}Z=-12.15 \\
p<.001 \\
E S=0.04\end{array}$ & $\begin{array}{c}Z=-10.61 \\
p<.001 \\
\text { ES }=0.08\end{array}$ & $\begin{array}{c}Z=-11.61 \\
p<.001 \\
\mathrm{ES}=0.06\end{array}$ \\
\hline Jungle Pellet (.28) & & $\begin{array}{c}Z=-\mathbf{3 . 9 5} \\
p<.001 \\
\mathrm{ES}=\mathbf{0 . 3 6}\end{array}$ & $\begin{array}{c}Z=-9.58 \\
p<.001 \\
\mathrm{ES}=0.07\end{array}$ & $\begin{array}{c}Z=-8.01 \\
p<.001 \\
\text { ES }=0.14\end{array}$ & $\begin{array}{c}Z=-9.03 \\
p<.001 \\
\text { ES }=1.07\end{array}$ \\
\hline Carrot (.10) & & & $\begin{array}{c}Z=-5.81 \\
p<.001 \\
\mathrm{ES}=0.20\end{array}$ & $\begin{array}{c}Z=-4.18 \\
p<.001 \\
\mathrm{ES}=0.40\end{array}$ & $\begin{array}{c}Z=-5.24 \\
p<.001 \\
\mathrm{ES}=\mathbf{0 . 3 0}\end{array}$ \\
\hline Celery (.02) & & & & $\begin{array}{c}Z=1.68 \\
p=.09 \\
\mathrm{ES}=2.00\end{array}$ & $\begin{array}{c}Z=0.59 \\
p=.55 \\
\mathrm{ES}=1.50\end{array}$ \\
\hline Toasted Oat (.04) & & & & & $\begin{array}{c}Z=-1.09 \\
p=.27 \\
\mathrm{ES}=0.75\end{array}$ \\
\hline
\end{tabular}


Huskisson et al. 96

\begin{tabular}{|c|c|c|c|c|c|}
\hline \multicolumn{6}{|l|}{ Nagoya (F, 2) } \\
\hline & Jungle Pellet (.24) & Carrot (.14) & Celery (.02) & Toasted Oat (.04) & Green Bean (.07) \\
\hline Peanut (.50) & $\begin{array}{c}Z=-\mathbf{3 . 2 3} \\
p=.001 \\
\mathrm{ES}=\mathbf{0 . 4 8}\end{array}$ & $\begin{array}{c}Z=-\mathbf{5 . 4 3} \\
p<.001 \\
\mathrm{ES}=\mathbf{0 . 2 8}\end{array}$ & $\begin{array}{c}Z=-13.67 \\
p<.001 \\
\text { ES }=0.04\end{array}$ & $\begin{array}{c}Z=-10.32 \\
p<.001 \\
E S=0.08\end{array}$ & $\begin{array}{c}Z=-7.75 \\
p<.001 \\
\text { ES }=0.14\end{array}$ \\
\hline Jungle Pellet (.24) & & $\begin{array}{c}Z=-2.25 \\
p=.02 \\
\mathrm{ES}=1.67\end{array}$ & $\begin{array}{c}Z=-10.72 \\
p<.001 \\
\mathrm{ES}=0.08\end{array}$ & $\begin{array}{c}Z=-7.31 \\
p<.001 \\
\mathrm{ES}=0.17\end{array}$ & $\begin{array}{c}Z=-4.65 \\
p<.001 \\
\mathrm{ES}=0.29\end{array}$ \\
\hline Carrot (.14) & & & $\begin{array}{c}Z=-\mathbf{8 . 6 3} \\
p<.001 \\
\mathrm{ES}=0.14\end{array}$ & $\begin{array}{c}Z=-5.15 \\
p<.001 \\
\mathrm{ES}=0.29\end{array}$ & $\begin{array}{c}Z=-\mathbf{2 . 4 3} \\
p=.02 \\
\mathrm{ES}=0.50\end{array}$ \\
\hline Celery (.02) & & & & $\begin{array}{c}Z=\mathbf{3 . 6 6} \\
p<.001 \\
\mathbf{E S}=\mathbf{0 . 5 0}\end{array}$ & $\begin{array}{c}Z=\mathbf{6 . 3 3} \\
p<.001 \\
\mathbf{E S}=\mathbf{3 . 5 0}\end{array}$ \\
\hline Toasted Oat (.04) & & & & & $\begin{aligned} Z & =2.75 \\
p & =.01 \\
\mathrm{ES} & =1.75\end{aligned}$ \\
\hline \multicolumn{6}{|l|}{ Otaru $(\mathrm{F}, 2)$} \\
\hline Peanut (.49) & $\begin{array}{c}Z=\mathbf{- 3 . 4 3} \\
p<.001 \\
\mathrm{ES}=\mathbf{0 . 4 7}\end{array}$ & $\begin{array}{c}Z=-5.04 \\
p<.001 \\
\mathrm{ES}=\mathbf{0 . 3 1}\end{array}$ & $\begin{array}{c}Z=-\mathbf{- 1 2 . 8 8} \\
p<.001 \\
\mathrm{ES}=\mathbf{0 . 0 4}\end{array}$ & $\begin{array}{c}Z=-\mathbf{7 . 9 5} \\
p<.001 \\
\mathrm{ES}=\mathbf{0 . 1 4}\end{array}$ & $\begin{array}{c}Z=\mathbf{- 9 . 5 9} \\
p<.001 \\
\mathrm{ES}=\mathbf{0 . 0 8}\end{array}$ \\
\hline Jungle Pellet (.23) & & $\begin{array}{c}Z=-1.73 \\
p=.08 \\
\mathrm{ES}=0.65\end{array}$ & $\begin{array}{c}Z=-\mathbf{9 . 7 9} \\
p<.001 \\
\mathrm{ES}=0.09\end{array}$ & $\begin{array}{c}Z=-4.73 \\
p<.001 \\
\text { ES }=0.30\end{array}$ & $\begin{array}{c}Z=-6.53 \\
p<.001 \\
\mathrm{ES}=0.17\end{array}$ \\
\hline Carrot (.15) & & & $\begin{array}{c}Z=\mathbf{- 8 . 1 7} \\
p<.001 \\
\mathrm{ES}=\mathbf{0 . 1 3}\end{array}$ & $\begin{array}{c}Z=-\mathbf{3 . 0 2} \\
p=.002 \\
\mathrm{ES}=\mathbf{0 . 4 7}\end{array}$ & $\begin{array}{c}Z=4.85 \\
p<.001 \\
\text { ES }=0.27\end{array}$ \\
\hline Celery (.02) & & & & $\begin{array}{c}Z=5.27 \\
p<.001 \\
\mathbf{E S}=\mathbf{3 . 5 0}\end{array}$ & $\begin{array}{c}Z=\mathbf{3 . 4 5} \\
p<.001 \\
\mathbf{E S}=\mathbf{2 . 0 0}\end{array}$ \\
\hline Toasted Oat (.07) & & & & & $\begin{array}{c}Z=-1.86 \\
p=.06 \\
\mathrm{ES}=0.57\end{array}$ \\
\hline
\end{tabular}

